\title{
Feynman integrals as A-hypergeometric functions
}

\author{
Leonardo de la Cruz \\ Higgs Centre for Theoretical Physics, School of Physics and Astronomy, \\ The University of Edinburgh, \\ Edinburgh EH9 3FD, Scotland, U.K. \\ E-mail: leonardo.delacruz@ed.ac.uk
}

ABSTRACT: We show that the Lee-Pomeransky parametric representation of Feynman integrals can be understood as a solution of a certain Gel'fand-Kapranov-Zelevinsky (GKZ) system. In order to define such GKZ system, we consider the polynomial obtained from the Symanzik polynomials $g=\mathcal{U}+\mathcal{F}$ as having indeterminate coefficients. Noncompact integration cycles can be determined from the coamoeba - the argument mapping - of the algebraic variety associated with $g$. In general, we add a deformation to $g$ in order to define integrals of generic graphs as linear combinations of their canonical series. We evaluate several Feynman integrals with arbitrary non-integer powers in the propagators using the canonical series algorithm.

Keywords: Scattering Amplitudes, Differential and Algebraic Geometry, Perturbative QCD

ArXiv EPrint: 1907.00507 


\section{Contents}

1 Introduction 1

2 A-hypergeometric functions and their representations 3

2.1 Notation 3

2.2 Polynomials, varieties, and their coamoebas 4

$\begin{array}{lll}2.3 & \text { GKZ systems and A-hypergeometric functions } & 7\end{array}$

2.3.1 Euler-type integral solutions 8

2.4 A-hypergeometric canonical series 9

$\begin{array}{ll}\text { 2.4.1 } & \text { Algorithm (Saito-Sturmfels-Takayama) } \\ & 12\end{array}$

$\begin{array}{lll}2.5 & \text { Examples } & 12\end{array}$

2.5.1 Double integral Gauss hypergeometric function 13

$\begin{array}{ll}\text { 2.5.2 Single integral Gauss hypergeometric function } & 15\end{array}$

3 Feynman integrals as A-hypergeometric functions $\quad \mathbf{1 6}$

$\begin{array}{lll}3.1 & \text { Lee-Pomeransky representation of Feynman integrals } & 17\end{array}$

$\begin{array}{lll}3.2 & \text { Feynman integrals and canonical series } & 18\end{array}$

3.3 Examples of $\operatorname{co}(\mathrm{A})=0 \quad 22$

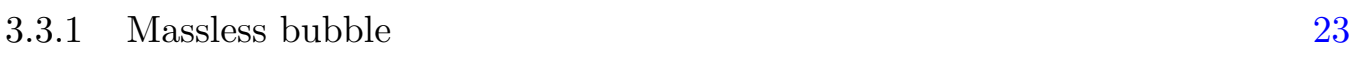

3.3.2 The single-scale massless triangle graph 24

$\begin{array}{ll}3.3 .3 & \text { The massless } L \text {-loop cantaloupe graph }\end{array}$

3.4 Examples of $\operatorname{co}(\mathrm{A})=1 \quad 28$

3.4.1 One-mass bubble 28

$\begin{array}{lll}3.4 .2 & \text { One-mass sunset } & 29\end{array}$

$\begin{array}{lll}3.4 .3 & \text { Single scale party hat } & 31\end{array}$

3.4.4 On-shell massless box $\quad 32$

$3.5 \quad \mathrm{~A} \operatorname{co}(\mathrm{A})=2$ example 34

4 Conclusions and outlook $\quad 36$

$\begin{array}{ll}\text { A Useful formulas } & 37\end{array}$

$\begin{array}{ll}\text { B Macaulay2 example } & 38\end{array}$

\section{Introduction}

The analytic evaluation of Feynman integrals in dimensional regularization is still one of the main challenges to compute higher order corrections to observables in collider experiments. Methods for evaluating Feynman integrals involve a good understanding of 
their analytic properties. These have been important from the very beginning in order to develop techniques to evaluate them. Long time ago, some of these properties led to the recognition that Feynman integrals satisfy systems of differential equations analogous to those of hypergeometric functions. ${ }^{1}$ The modern method to evaluate Feynman integrals is indeed based on differential equations. It is the combination of Integration by Parts (IBP) identities and differential equations [3-8]. The result is particularly simple [9] if the system of differential equations evaluates to combinations of Multiple Polylogarithms [10-13].

In favorable cases, Feynman integrals can be evaluated in terms of classical hypergeometric functions and their generalizations such as Appell, Horn, and Lauricella hypergeometric functions. Typically, these functions appear as infinite sums through the Mellin-Barnes representation of Feynman integrals (see e.g., [14-16]). Once evaluated in terms of hypergeometric functions, they can be utilized to recover the $\epsilon$-expansion [17-20], although it is a nontrivial task when hypergeometric functions of many variables appear (see e.g., [21, 22]). More recently, the Mellin-Barnes representation has been used to find systems of differential equations without resorting to IBP identities [23], through the differential reduction method [17, 23-30], which is based on refs. [31, 32]. Using the Mellin-Barnes representation it has been shown that a large class of Feynman integrals can be expressed in terms of Horn-type functions [33]. The differential reduction approach has led to the application of techniques coming from $D$-module theory as has been recently shown in ref. [34]. This point of view is close to the one we will adopt in this paper.

A-hypergeometric functions were introduced by Gel'fand-Kapranov-Zelevinsky (GKZ) in 1990 [35] as a generalization of the well-known Appell, Lauricella, and Horn series. One important aspect of the theory is the study of polynomials with indeterminate coefficients associated with an integer matrix $A$ [36]. This matrix and a vector of complex parameters furnish a system of partial differential equations (PDEs) known as a GKZ system. Solutions of these systems of PDEs are called A-hypergeometric functions. They can be represented as Euler-type integrals and hence these integrals define A-hypergeometric functions [35, 3739]. On the other hand, series solutions can be computed by a generalization of the Frobenius method known as the canonical series algorithm due to Saito, Sturmfels, and Takayama [40]. GKZ systems and Feynman integrals have been object of recent interest to mathematicians, who have studied the relation among GKZ systems, Feynman integrals, and their regularization [41, 42]. Maximal cuts in this language have been studied in ref. [43].

In this paper, we will consider the parametric representation of Feynman integrals employed by Lee and Pomeransky to relate critical points of the sum of the Symanzik polynomials and the number of master integrals arising from IBPs [44]. This representation is based on the polynomial $g=\mathcal{U}+\mathcal{F}$, where $\mathcal{U}$ and $\mathcal{F}$ are the first and second Symanzik polynomials, respectively. We will show that the polynomial $g$ defines a GKZ system, which is constructed by considering its coefficients to be indeterminate. We will use this information to obtain series expansions of the Euler integrals solutions using the SaitoSturmfels-Takayama canonical series algorithm. Our definition of a GKZ system based on $g$

\footnotetext{
${ }^{1}$ It is due to Regge the conjecture that Feynman integrals belong to a generalization hypergeometric functions [1] and hence that Feynman integrals satisfy analogous systems of differential equations. See ref. [2].
} 
allows the evaluation of integrals with arbitrary noninteger powers in the propagators using computational algebra. The polynomial $g$ may lead to a matrix of codimension 0 . In such cases, we introduce a deformation of $g$ to ensure a canonical series representation. Once the canonical series are computed and integration constants are obtained, Feynman integrals can be recovered at the end of the computation by taking the limit of the deformation going to zero and setting its coefficients to their kinematic values.

This paper is organized as follows: in section 2 we review the GKZ framework, present their solutions, and introduce the canonical series algorithm. We end this section with examples. In section 3 we introduce scalar Feynman integrals and furnish a GKZ system based on them suited for canonical series. We give examples at the end of section 3 . We give our conclusions in section 4.

\section{A-hypergeometric functions and their representations}

In this section, we review basic aspects of the GKZ approach to hypergeometric functions. The main references are the book [40] and the lectures [45]. Reviews of the main concepts can also be found in refs. [46, 47].

This review contains four main parts. We first introduce polynomials with indeterminate coefficients (toric polynomials), which is one of the main ideas behind the GKZ approach. Polynomials with indeterminate coefficients lead to generalizations of discriminants, resultants, and determinants through the study of toric varieties ${ }^{2}$ [36].

In the second part, we associate a system of partial differential equations (PDEs) to polynomials with indeterminate coefficients. We introduce Euler integral representations of solutions of GKZ systems. The system of PDEs may be formally defined as a holonomic ideal in a Weyl algebra $D$ and it is the proper language for computational algebra purposes [40].

In the third part, we introduce series representations of solutions of GKZ systems and review the Saito-Sturmfels-Takayama algorithm to compute canonical series [40]. The fourth part contains examples of the methods introduced. A short example using the computer algebra system Macaulay2 can be found in appendix B.

The connection with Feynman integrals will be made in section 3. The Saito-SturmfelsTakayama algorithm will be our main tool to evaluate Feynman integrals in section 3.

\subsection{Notation}

Throughout this paper we will employ multi-index notation, i.e.,

$$
z^{\alpha}:=z_{1}^{\alpha_{1}} \cdots z_{N}^{\alpha_{N}}, \quad c^{\gamma}:=c_{1}^{\gamma_{1}} \cdots c_{n}^{\gamma_{n}}
$$

where $\alpha \in \mathbb{K}^{N}, \gamma \in \mathbb{K}^{n}$. For polynomials $b_{1}(z), \ldots, b_{M}(z)$ in the variables $z=\left(z_{1}, \ldots, z_{N}\right)$, the multi-index notation for products of polynomials reads

$$
b(z)^{\beta}:=b_{1}(z)^{\beta_{1}} b_{2}(z)^{\beta_{2}} \cdots b_{M}(z)^{\beta_{M}},
$$

\footnotetext{
${ }^{2}$ An algebraic toric variety is of the form $\mathbb{C}_{*}^{n}$, where $\mathbb{C}_{*}=\mathbb{C} \backslash\{0\}$.
} 
where $\beta \in \mathbb{K}^{M}$. Typically we will have $\mathbb{K}=\mathbb{C}$. For $\beta=(1, \ldots, 1)$, we simply write $b(z)^{(1, \ldots, 1)}=b(z)$. In the case $M=1$, we write $b_{1}(z)^{\beta_{1}}=b(z)^{\beta}$. We call polynomials with indeterminate coefficients toric polynomials ${ }^{3}$ and emphasize their dependence on their coefficients $c$ by writing $b(c, z)$. The multi-index notation for differential operators reads $\partial^{\alpha}=\partial_{1}^{\alpha_{1}}, \cdots \partial_{n}^{\alpha_{n}}$, where $\partial_{i}=\partial / \partial c_{i}$. Euler operators are defined by

$$
\theta_{i}:=c_{i} \frac{\partial}{\partial c_{i}}=c_{i} \partial_{i}
$$

Integrals where toric polynomials are involved will be identified by a subscript, e.g., $I_{b}$ indicates that the integral under consideration has a toric polynomial $b(c, z)$ on its integrand. Finally, the Pochhammer symbol is defined by

$$
(a)_{n}:=\frac{\Gamma(a+n)}{\Gamma(a)}, \quad a \in \mathbb{C} \backslash \mathbb{Z}_{\leq 0} .
$$

Useful identities following from this definition are summarized in appendix A.

\subsection{Polynomials, varieties, and their coamoebas}

Let us consider $q$ Laurent polynomials in $N$ variables of the form

$$
b_{i}(z)=\sum_{j=1}^{n_{i}} c_{i j} z^{\alpha_{i j}}, \quad c_{i j} \in \mathbb{C}_{*}, \quad i=1, \ldots, q,
$$

where $\mathbb{C}_{*}=\mathbb{C} \backslash\{0\}, \alpha_{i j} \in \mathbb{Z}^{N}$, and $n_{i}$ is the length of the set of exponent vectors $A_{i}=$ $\left\{\alpha_{i 1}, \cdots, \alpha_{i k}, \cdots, \alpha_{i n_{i}}\right\}$ associated with the polynomial $b_{i}(z)$. By abuse of notation, we denote by $A_{i}$ the $N \times n_{i}$ configuration matrix of the exponent vectors of the $i$-th polynomial as

$$
A_{i}=\left(\begin{array}{lllll}
\alpha_{i 1} & \cdots & \alpha_{i k} & \cdots & \alpha_{i n_{i}}
\end{array}\right), \quad \alpha_{i k} \in \mathbb{Z}^{N},
$$

where each (column) vector $\alpha_{i k}$ is associated with a monomial term $c_{i k} z^{\alpha_{i k}}$ in $b_{i}(z)$. Therefore $\left|A_{i}\right|=n_{i}$ is the total number of monomials (columns in $\left.A_{i}\right)^{4}{ }^{4}$

Let us give an example. Suppose we have a single polynomial in $N$ variables with $n$ monomial terms $b^{\mathrm{ex}}(z)=\sum_{j=1}^{n} c_{j} z^{\alpha_{j}}$, where we have labeled the coefficients simply by $c_{j}$, $j=1, \ldots, n$. The $N \times n$ configuration matrix of $b^{\mathrm{ex}}(z)$ reads

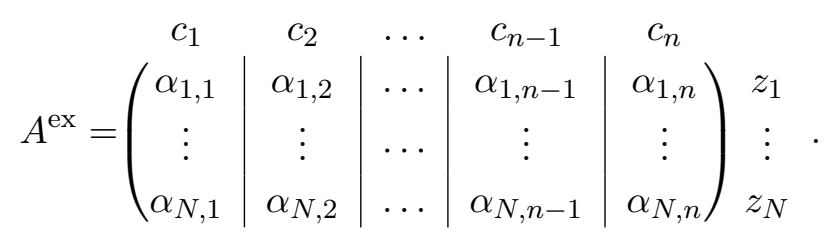

\footnotetext{
${ }^{3}$ The reason of this nomenclature is that polynomials with indeterminate coefficients will be associated with an integer matrix, which can be interpreted as representing a toric variety.

${ }^{4}$ Here we are adopting a rather unusual route by defining first Laurent polynomials and then the matrices $A_{i}$. The reason behind this is that in Feynman integrals we first consider polynomials with determinate coefficients and then consider the indeterminate case. In the mathematics literature, typically one first considers a configuration matrix and associates a polynomial to it.
} 
The monomial term related with the first column reads

$$
c_{1} z^{\alpha_{1}}=c_{1} z_{1}^{\alpha_{1,1}} \cdots z_{N}^{\alpha_{N, 1}} .
$$

With the above identifications of the columns and rows, the arrangement of the rows and columns (terms) is irrelevant as they define the same polynomial.

Let us now consider the product

$$
b(z):=b_{1}(z) \cdots b_{q}(z),
$$

where each polynomial and its configuration $A_{i}$ are taken independently, in other words we do not expand $b(z)$. Expanding the polynomials would lead to a single polynomial and hence it is a special case of the above. Let $n:=n_{1}+\cdots+n_{q}$ be the total number of monomials and let us define the $(N+q) \times n$ matrix

$$
\mathrm{A}:=\left(\begin{array}{cccc}
1 & 0 & \ldots & 0 \\
0 & 1 & \ldots & 0 \\
\vdots & \vdots & \ddots & \vdots \\
0 & 0 & \ldots & 1 \\
A_{1} & A_{2} & \ldots & A_{q}
\end{array}\right)
$$

associated with $b(z)$. Here $0=(0, \ldots, 0)$ and $1=(1, \ldots, 1)$ are row vectors of length $\left|A_{i}\right|$. We define the codimension of $A$ as

$$
\operatorname{co}(\mathrm{A}):=n-N-q .
$$

The matrix $A$ is the main object of study of the GKZ approach to hypergeometric functions. This definition allows us to consider integrals and PDEs later. This matrix is interpreted as representing a toric variety. ${ }^{5}$

For later purpose we introduce the Newton polytopes of $b_{i}(z)$ following ref. [38]. For each $b_{i}(z)$, the Newton polytope is the convex hull $\Delta_{b_{i}}=\operatorname{conv}\left(\alpha_{i 1}, \ldots, \alpha_{i n_{i}}\right)$ in $\mathbb{R}^{N}$. Like any other polytope, we can represent $\Delta_{b_{i}}$ as the intersection of a finite number of halfspaces: ${ }^{6}$

$$
\Delta_{b_{i}}=\bigcap_{j=1}^{M_{i}}\left\{\sigma \in \mathbb{R}^{N}: \mu_{j}^{i} \cdot \sigma \geq \nu_{j}^{i}\right\},
$$

where $\mu_{j}^{i} \in \mathbb{Z}^{N}$ are primitive integer vectors in the inward normal direction of the facets of $\Delta_{b_{i}}$, and the $\nu_{j}^{i} \in \mathbb{Z}$ are integers (see figure 1). Here the product $X \cdot Y$ stands for the standard scalar product in $\mathbb{R}^{N}$. The polytope of $b(z)$ is the Minkowski sum ${ }^{7}$

$$
\Delta_{b}=\Delta_{b_{1} b_{2} \cdots b_{q}}=\Delta_{b_{1}}+\cdots+\Delta_{b_{q}} .
$$

\footnotetext{
${ }^{5}$ See ref. [48] for an introduction to toric ideals.

${ }^{6}$ Any polytope has a vertex representation and a half space representation. Going from one representation to another involves conversion algorithms that are beyond the scope of the present work. The interested reader can consult e.g., ref. [49].

${ }^{7}$ For two polytopes $P$ and $Q$ in $\mathbb{R}^{N}$, their Minkowski sum $P+Q$ is the set of all vectors $p+q$, such that $p \in P, q \in Q$ [49].
} 


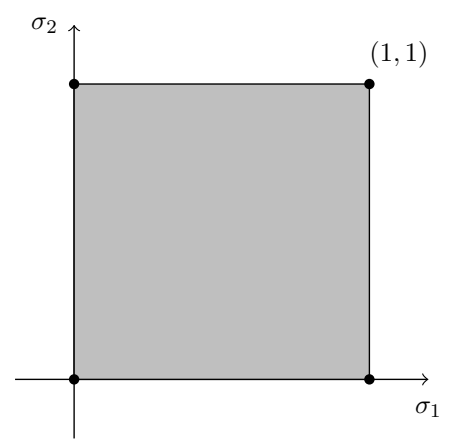

Figure 1. Polytope of $b(z)=1+z_{1}+z_{2}+c z_{1} z_{2}$. Written as intersection of half planes, we have $\Delta_{b}=\left\{\sigma_{1} \geq 0\right\} \cap\left\{\sigma_{2} \geq 0\right\} \cap\left\{-\sigma_{1} \geq-1\right\} \cap\left\{-\sigma_{2} \geq-1\right\}$.

Let us now review the concept of a coamoeba of a variety. Consider the ideal $I$ generated by some polynomials $f_{1}, \ldots, f_{j} \subset \mathbb{C}\left[z_{1}, \ldots, z_{N}\right]$. The zero set of the ideal $I=\left\langle f_{1}, \ldots, f_{j}\right\rangle$ defines the algebraic variety

$$
\mathcal{V}(I):=\left\{z \in \mathbb{C}^{N}: f_{i}(z)=0, \text { for all } f_{i} \in I\right\}
$$

Let us consider the case of a single polynomial $f$. The amoeba $\mathcal{A}_{f}$ of the algebraic variety $\mathcal{V}(f)$ is the image of $\mathcal{V}(f)$ under the log mapping

$$
\mathcal{A}_{f}:=\log (\mathcal{V}(f))
$$

where $\log (z)=\left(\log \left|z_{1}\right|, \ldots, \log \left|z_{N}\right|\right)$. Amoebas were introduced by GKZ in ref. [36]. Similarly, the coamoeba of $\mathcal{V}(f)$ is the image of $\mathcal{V}(f)$ under the argument mapping

$$
\mathcal{A}_{f}^{\prime}:=\operatorname{Arg}(\mathcal{V}(f))
$$

where $\operatorname{Arg}(z)=\left(\arg \left(z_{1}\right), \ldots, \arg \left(z_{N}\right)\right)$. Here $\arg (z)$ is defined as usual. For example, the real positive line is given by $\mathbb{R}_{+}=\operatorname{Arg}^{-1}(0)$.

Unlike the log mapping, the argument mapping is multivalued and we can think of it as a multiple periodic subset of $\mathbb{R}^{N}$ or equivalently it can be viewed as the $N$-dimensional algebraic torus $\mathbb{T}^{N}=(\mathbb{R} / 2 \pi \mathbb{Z})^{N}$ [38]. Coamoebas were introduced by Passare in $2004 .^{8}$ Our motivation to introduce coamoebas is that they define noncompact integration cycles for Euler-Mellin integral representations of A-hypergeometric functions. These cycles are particularly useful in generic cases where polynomials may vanish on the integration region. In many cases, coamoebas are difficult to study analytically and one has to consider a rough version, which is called the lopsided coamoeba [52-55]. The relation between Ahypergeometric functions and coamoebas has been studied in refs. [37, 38, 55, 56]. See refs. [46, 52] for an introduction to coamoebas.

\footnotetext{
${ }^{8}$ Coamoebas have appeared later in the physics literature under the name of algae in the context of dimer models, see e.g., [50, 51].
} 


\subsection{GKZ systems and A-hypergeometric functions}

We denote by $H_{\mathrm{A}}(\kappa)$ the GKZ system associated with a matrix A and a vector of parameters $\kappa$. It is defined by the following data:

1. A $(N+q) \times n$ matrix $\mathrm{A}$ such that the vector $(1, \ldots, 1)$ lies in its row span. Hence, there is a vector $\xi \in \mathbb{Z}^{N+q}$, such that $\xi \mathrm{A}=(1, \cdots, 1)$. By definition this matrix is obtained from

$$
b(z)=b_{1}(z) \cdots b_{q}(z)
$$

2. A system of partial differential equations (PDEs) associated with A. Let $u, v \in \mathbb{N}^{n}$ and consider

$$
\begin{aligned}
\left(\partial^{u}-\partial^{v}\right) F(c) & =0, \quad \text { where } \quad \mathrm{A} u=\mathrm{A} v \\
\left(\sum_{j=1}^{n} a_{i j} \theta_{j}-\kappa_{i}\right) F(c) & =0, \quad i=1, \ldots, N+q
\end{aligned}
$$

where $a_{i j}$ denotes the components of A. Recall that $\theta_{j}=c_{j} \partial / \partial c_{j}$ and $\partial^{u}=\partial_{1}^{u_{1}} \cdots \partial_{n}^{u_{n}}$.

3. A vector of parameters $\kappa=\left(\kappa_{1}, \ldots, \kappa_{N+q}\right), \kappa_{i} \in \mathbb{K}$.

A holomorphic function $F(c)$ or formal series is called A-hypergeometric if it satisfies the above system of PDEs.

GKZ systems can be rigorously defined in the language of holonomic ideals in the ring of differential operators with polynomial coefficients - the so called Weyl algebra $D=\mathbb{K}\left\langle c_{1}, \ldots, c_{n}, \partial_{1}, \ldots, \partial_{n}\right\rangle$ modulo commutation rules. In this sense, the toric ideal associated with $\mathrm{A}$ is defined by

$$
I_{\mathrm{A}}:=\left\langle\partial^{u}-\partial^{v}: \mathrm{A} u=\mathrm{A} v, \quad u, v \in \mathbb{N}^{n}\right\rangle \subset \mathbb{K}\left[\partial_{1}, \ldots, \partial_{n}\right],
$$

where $\mathbb{K}\left[\partial_{1}, \ldots, \partial_{n}\right]$ is a commutative polynomial ring. In addition, we construct the ideal generated by the column vectors $\kappa^{T}$ and $\theta=\left(\theta_{1}, \ldots, \theta_{n}\right)^{T}$. This ideal is given by

$$
\left\langle\mathrm{A} \theta-\kappa^{T}\right\rangle \subset \mathbb{K}\left[\theta_{1}, \ldots, \theta_{n}\right],
$$

where each generator of the ideal has the form $\sum_{j=1}^{n} a_{i j} \theta_{j}-\kappa_{i}$. The GKZ system $H_{\mathrm{A}}(\kappa)$ denotes the left ideal on the Weyl algebra $D$ generated by $I_{\mathrm{A}}$ and $\left\langle\mathrm{A} \theta-\kappa^{T}\right\rangle$. In this language a holomorphic function $F(c)$ or formal series is called A-hypergeometric of degree $\kappa$ if $H_{\mathrm{A}}(\kappa) \bullet F(c)=0$, where $\bullet$ denotes the action of the Weyl algebra on polynomials [40]. The language of holonomic ideals and $D$-modules is the appropriate one to treat the problem using computational algebra.

Let us denote by $\operatorname{vol}(\mathrm{A})$ the normalized volume - w.r.t. the volume of the standard simplex which is equal to 1 - of the convex hull of A. ${ }^{9}$ Then, for generic parameters ${ }^{10} \kappa$,

\footnotetext{
${ }^{9}$ For the case $q=1$, this normalization implies that $\operatorname{vol}(\mathrm{A})=N ! \operatorname{vol}\left(\Delta_{b}\right)$ with $\Delta_{b}$ defined in section 2.2 .

${ }^{10}$ Here, generic parameters $\kappa \in \mathbb{K}^{N+q}$ are to be understood as ranging over nonempty open algebraic (Zariski) subsets of $\mathbb{K}^{N+q}$.
} 
the rank of the system satisfies the inequality (Theorem 3.5.1 in [40])

$$
\operatorname{rank}\left(H_{\mathrm{A}}(\kappa)\right) \geq \operatorname{vol}(\mathrm{A})
$$

which corresponds to the dimension of the solution space. This number can also be computed from the degree of the toric ideal $I_{\mathrm{A}}$.

For generic parameters $\operatorname{rank}\left(H_{\mathrm{A}}(\kappa)\right)=\operatorname{vol}(\mathrm{A})$. In cases where the parameters have certain special values (nongeneric) the rank of the system can jump and we have $\operatorname{rank}\left(H_{\mathrm{A}}(\kappa)\right)>\operatorname{vol}(\mathrm{A})$ (example 4.2.7 in ref. [40]).

\subsubsection{Euler-type integral solutions}

Solutions of GKZ systems have representations as Euler-type integrals. Accordingly, we call them A-hypergeometric functions. They can be constructed by taking the vector of parameters

$$
\kappa=(-\beta,-\alpha), \quad \beta \in \mathbb{C}^{q}, \quad \alpha \in \mathbb{C}^{N},
$$

and then write the integral associated with $A$ as follows:

$$
I_{b}(\kappa)=\int_{\Omega} \frac{z^{\alpha}}{b(c, z)^{\beta}} \mathrm{d} \eta_{N}, \quad \mathrm{~d} \eta_{N}=\frac{d z_{1}}{z_{1}} \wedge \frac{d z_{2}}{z_{2}} \wedge \cdots \wedge \frac{d z_{N}}{z_{N}},
$$

where the integration cycle is such that $\Omega \subset\left(\mathbb{C}_{*}\right)^{N} \backslash \mathcal{V}(b)$. It is usually assumed that the cycles are compact [35].

In ref. [38], Berkesh, Forsgård, and Passare (BFP) constructed explicit noncompact cycles for these type integrals. In order to reach the above type of integral, BFP proceed in three steps. First, we consider that $b(z)$ does not vanish in the positive orthant and consider the Euler-Mellin integral

$$
I(\kappa)=\int_{\mathbb{R}_{+}^{N}} \frac{z^{\alpha}}{b(z)^{\beta}} \mathrm{d} \eta_{N}=\int_{\mathbb{R}^{N}} \frac{e^{(\alpha, x)}}{b\left(e^{x}\right)^{\beta}} \mathrm{d} x, \quad \mathrm{~d} x=d x_{1} \wedge \cdots \wedge d x_{N},
$$

where in this case polynomial coefficients are fixed. BFP showed that if the polynomials $b(z)$ are nonvanishing, ${ }^{11}$ then eq. (2.20) converges and defines and analytic funcion with parameters $\kappa=(-\beta,-\alpha)$ on the tube domain

$$
\left\{(\alpha, \beta) \in \mathbb{C}^{N+q} \mid \tau:=\operatorname{Re} \beta \in \mathbb{R}_{+}^{q}, \quad \sigma:=\operatorname{Re} \alpha \in \operatorname{int}\left(\tau \Delta_{b}\right)\right\},
$$

where $\operatorname{int}\left(\tau \Delta_{b}\right)$ is the interior of of the weighted Minkowski sum of the Newton polytopes of $b_{j}$ weighted by $\tau$, i.e., $\tau \Delta_{b}=\sum_{j=1}^{q} \tau_{j} \Delta_{b_{j}}$. The second step is to consider the less favorable case where the polynomials $b(z)$ vanish on the positive orthant. Here, we can take a connected component $\Theta$ of $\mathbb{R}^{N} \backslash \overline{\mathcal{A}}_{b}^{\prime}$, where $\overline{\mathcal{A}}_{b}^{\prime}$ denotes the closure of the coamoeba of $b$ and consider the integral

$$
I(\kappa)=\int_{\operatorname{Arg}^{-1} \theta} \frac{z^{\alpha}}{b(z)^{\beta}} \mathrm{d} \eta_{N}=\int_{\mathbb{R}^{N}} \frac{e^{\alpha \cdot(x+\mathrm{i} \theta)}}{b\left(e^{x+\mathrm{i} \theta}\right)^{\beta}} \mathrm{d} x,
$$

\footnotetext{
${ }^{11}$ Technically, these polynomials should be completely nonvanishing in the sense of BFP i.e., not vanishing on the faces of the polytope of $b(z)$. See definition 2.1 of ref. [38]. See also [46].
} 


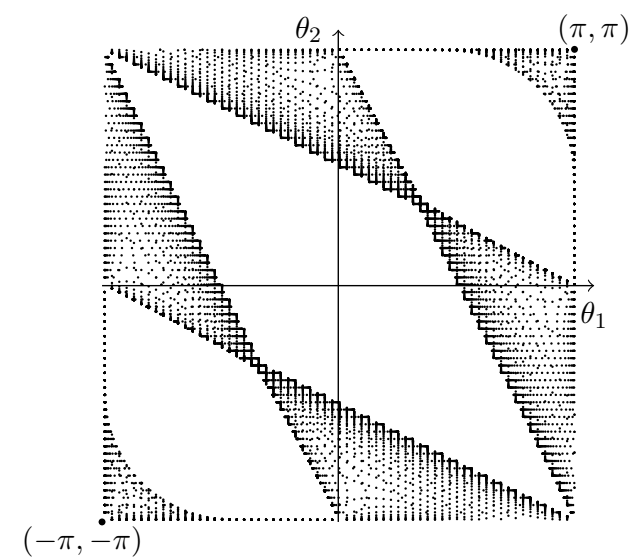

Figure 2. Coamoeba (shaded) of $b=1+z_{1} z_{2}+z_{1} z_{2}^{2}+z_{1}^{2} z_{2}^{2}+c_{4} z_{1}^{2} z_{2}$ drawn in the fundamental domain $[-\pi, \pi] \times[-\pi, \pi]$ of $\mathbb{T}^{2}$ in $\mathbb{R}^{2}$. This case corresponds to $c=\left(1,1,1,1, c_{4}\right)$, with $c_{4}$ near 1 . Here, we have three connected components, one of which contains $(0,0)$ (center of the figure).

where $\theta \in \Theta$ is a representative of the connected component of complement of coamoeba of $b(z)$. This is essentially a change of variables and a slight perturbation of $\theta$ does not impact the result of the integral. We write an analogue of Theorem 2.4 in ref. [38], where the proof can be found.

Theorem (Berkesh, Forsgård, and Passare). For nonvanishing polynomials, $b_{1}, \ldots, b_{q}$ in $\mathrm{Arg}^{-1} \theta$ the integral (2.22) admits a meromorphic continuation of the form

$$
I(\kappa)=\Phi_{b}^{\Theta}(\alpha, \beta) \prod_{k=1}^{M} \Gamma\left(\mu_{k} \cdot \alpha-\nu_{k} \cdot \beta\right),
$$

where $\Phi_{b}^{\Theta}(\alpha, \beta)$ is an entire function and $\Theta$ is a connected component. $\mu_{k}, \nu_{k}$ can be recovered from the Newton polytope $\Delta_{b_{1} \cdots b_{q}}$ (see eq. (2.7)).

The third step is the transition to A-hypergeometric functions by promoting the coefficients of $b(z)$ in eq. (2.22) to indeterminate, therefore we consider them as variables. The integral ${ }^{12}$

$$
I_{b}(\kappa)=\int_{\operatorname{Arg}^{-1} \theta} \frac{z^{\alpha}}{b(c, z)^{\beta}} \mathrm{d} \eta_{N}
$$

is a representation of an A-hypergeometric function (Theorem 4.2 in [38]). For generic parameters $\kappa$, eq. (2.24) provides a basis of solutions of $H_{\mathrm{A}}(\kappa)$, where each integral is evaluated on a representative of $\Theta$ for each connected component of $\mathbb{R}^{N} \backslash \overline{\mathcal{A}}_{b}^{\prime}$ (see figure $2^{13}$ ).

\subsection{A-hypergeometric canonical series}

Saito, Sturmfels, and Takayama (SST) generalized the Frobenius method using Gröbner deformations in order to deal with regular holonomic systems (chapters 2 and 3 of [40]).

\footnotetext{
${ }^{12}$ Here $c \in \mathbb{C}^{n} \backslash \Sigma_{\mathrm{A}}$, where $\Sigma_{\mathrm{A}}$ is the singular locus of all A-hypergeometric functions.

${ }^{13}$ We thank Jens Forsgård for providing his MATHEMATICA package to draw coamoebas and lopsided coamoebas.
} 
Roughly speaking, it consists on taking certain initial ideals of $I_{\mathrm{A}}$ (eq. (2.15)) with respect to a weight $w \in \mathbb{R}^{n}$ and generate the series solutions from it. The role of the indicial equation in the Frobenius method is played by an indicial ideal $\operatorname{ind}_{w}\left(I_{\mathrm{A}}\right)$ with respect to $w$, together with the ideal $\left\langle\mathrm{A} \theta-\kappa^{T}\right\rangle$. As in the Frobenius method, the problem consist of finding the roots $\gamma$ of those ideals and generate the coefficients of the series. Series thus obtained belong to the Nilsson ring, i.e, series of the form

$$
f=\sum_{\alpha, \beta} k_{\alpha \beta} c^{\alpha} \log (c)^{\beta}
$$

In this paper, we will be chiefly interested in the case where the resulting series are logarithm-free, i.e., where $\beta=0$ in the above equation. Let us turn our attention to these cases. Let

$$
\mathcal{L}:=\left\{u \in \mathbb{Z}^{n}: \mathrm{A} u=0\right\}
$$

be a lattice of rank $m$ and let $\kappa^{T}=\mathrm{A} \gamma^{T}{ }^{14}$ For $u \in \mathcal{L}$ we can write $u=u_{+}-u_{-}$, where $u_{ \pm} \in \mathbb{N}^{n}$ have disjoint support. Now, for $\gamma \in \mathbb{C}^{n}$ we define the following quantities expressed as falling factorials

$$
\begin{aligned}
{[\gamma]_{u_{-}} } & :=\prod_{i: u_{i}<0} \prod_{j=1}^{-u_{i}}\left(\gamma_{i}-j+1\right)=\prod_{i: u_{i}<0}(-1)^{-u_{i}}\left(\gamma_{i}\right)_{-u_{i}}, \\
{[\gamma+u]_{u_{+}} } & :=\prod_{i: u_{i}>0} \prod_{j=1}^{u_{i}}\left(\gamma_{i}+u_{i}-j+1\right)=\prod_{i: u_{i}>0} \prod_{j=1}^{u_{i}}\left(\gamma_{i}+j\right)=\prod_{i: u_{i}>0}\left(\gamma_{i}+1\right)_{u_{i}},
\end{aligned}
$$

where $(a)_{x}$ are Pochhammer symbols. Then for $\gamma \in \mathbb{C}^{n}$, such that no element in $\gamma$ is a nonnegative integer, the series

$$
\phi_{\gamma}:=\sum_{u \in \mathcal{L}} \frac{[\gamma]_{u_{-}}}{[\gamma+u]_{u_{+}}} c^{(\gamma+u)}
$$

is a solution of $H_{\mathrm{A}}(\kappa)$ (see proposition 3.4.1 and Theorem 3.4.2 in ref. [40]). When the vectors $\gamma$ contain negative integers, we define the negative support of $\gamma$ as

$$
\overline{\operatorname{supp}}(\gamma)=\left\{i \in\{1, \ldots, n\}: \gamma_{i} \in \mathbb{Z}_{<0}\right\} .
$$

In those cases, we consider the lattice

$$
\mathcal{N}_{\gamma}:=\{u \in \mathcal{L} \mid \overline{\operatorname{supp}}(\gamma+u)=\overline{\operatorname{supp}}(\gamma)\}
$$

and perform the sum over $\mathcal{N}_{\gamma}$ in eq. (2.29). In this paper, we will assume that the vectors $\kappa$ are generic and thus none of the roots $\gamma$ are negative integers. ${ }^{15}$ This implies that $\overline{\operatorname{supp}}(\gamma)=\emptyset$ and therefore the sum runs over $u \in \mathcal{L}$.

The roots $\gamma$ can be obtained by finding the roots of an ideal known as fake indicial ideal. Accordingly, the roots are called fake exponents and we give the algorithm to compute

\footnotetext{
${ }^{14}$ Notice that we will not be interested in general vectors $\gamma \in \mathbb{C}^{n}$ but only those which are the roots of an ideal of $H_{\mathrm{A}}(\kappa)$ with respect to some weight vector $w$. See Algorithm below.

${ }^{15}$ Corollary 3.4 .3 in ref. [40].
} 
them below. Series thus obtained are called canonical series. The series obtained from this algorithm have a common domain of convergence $\mathcal{U}_{w} \in \mathbb{C}^{n}$, which is characterized by a weight vector $w$. For generic roots $\gamma$, canonical series provide a basis of holomorphic solutions of $H_{\mathrm{A}}(\kappa)$ in $\mathcal{U}_{w}$ (for a description of this domain see Theorem 2.5.16 in ref. [40] and Theorem 3.19 in ref. [45]).

The relation with Euler integrals goes as follows. Suppose we find $r$ different roots. The general solution of the integral (2.19) is given by a linear combination of its canonical series [40], i.e.,

$$
I_{b}(\kappa)=K_{1} \phi_{1}+\cdots+K_{r} \phi_{r}
$$

where the canonical series $\phi_{r}$ can be computed independently of the cycle $\Omega$. The integration cycle plays a role in the computation of the integration constants $K_{i}$. We discuss how to obtain integrations constants for the particular case of Feynman integrals in the next section.

We proceed with the canonical series algorithm to compute fake exponents $\gamma$. A comprehensive review of this algorithm can be found in ref. [45]. We start with some definitions.

\section{Definitions.}

Toric ideal. Let $D$ be a Weyl algebra over $\mathbb{K}$, which is a free (noncommutative) associative $\mathbb{K}$-algebra generated by $\mathbb{K}\left\langle c_{1}, \ldots, c_{n}, \partial_{1}, \ldots, \partial_{n}\right\rangle$ modulo the commutation rules

$$
c_{i} c_{j}=c_{j} c_{i}, \quad \partial_{i} \partial_{j}=\partial_{j} \partial_{i}, \quad \partial_{i} c_{j}=\delta_{i j} .
$$

Let $\mathrm{A}$ be as in eq. (2.5), then

$$
I_{\mathrm{A}}:=\left\langle\partial^{u}-\partial^{v}: \mathrm{A} u=\mathrm{A} v, \quad u, v \in \mathbb{N}^{n}\right\rangle
$$

generates the toric ideal associated with A. Toric ideals can be computed using reduced Gröbner bases [57].

Initial ideal. Let $w \in \mathbb{R}^{n}$ be a weight vector and let $I_{\mathrm{A}}$ be a toric ideal. For $w$ generic, the ideal $\operatorname{in}_{w}\left(I_{A}\right)$ is a monomial ideal generated by the leading terms of $I_{A}$ with respect to the partial ordering $\preceq_{w}$.

Standard pairs. Let $R=\mathbb{K}\left[\partial_{1}, \ldots, \partial_{n}\right]$ and let $I$ be a monomial ideal in $R$. Furthermore, let $\partial^{\alpha}$ be a monomial and $F \subseteq\{1, \ldots, n\}$, where $\alpha \in \mathbb{N}^{n}$. A standard pair of a monomial ideal $I$ is a pair $\left(\partial^{\alpha}, F\right)$ satisfying three conditions:

1. $\alpha_{i}=0$ for all $i \in F$,

2. for all choices of integers $\beta_{j} \geq 0$, the monomial $\partial^{\alpha} \prod_{j \in F} \partial_{j}^{\beta_{j}} \notin I$,

3. for all $l \notin F$, there exist $\beta_{j} \geq 0$ such that $\partial^{\alpha} \partial_{l}^{\beta_{l}} \prod_{j \in F} \partial_{j}^{\beta_{j}} \in I$.

Let us denoted by $\mathcal{S}(I)$ the set of all standard pairs of $I$. The decomposition of $I$ into irreducible monomial ideals can be obtained from the identity.

$$
I=\bigcap_{\left(\partial^{\alpha}, F\right) \in \mathcal{S}(I)}\left\langle\partial_{i}^{\alpha_{i}+1}: i \in F\right\rangle .
$$




\subsubsection{Algorithm (Saito-Sturmfels-Takayama)}

In this algorithm we will set $\mathbb{K}=\mathbb{C}$.

Input: matrix $\mathrm{A}$, weight vector $w$, and complex parameters $\kappa$.

Output: roots of the fake indicial ideal $\operatorname{fin}_{w}\left(H_{\mathrm{A}}(\kappa)\right)$.

1. Compute the toric ideal associated with $A$

$$
I_{\mathrm{A}}=\left\langle\partial^{u}-\partial^{v}: \mathrm{A} u=\mathrm{A} v, \quad u, v \in \mathbb{N}^{n}\right\rangle .
$$

Notice that this is an ideal in the commutative polynomial ring $\mathbb{C}\left[\partial_{1}, \ldots, \partial_{n}\right]$. This ideal is the input to compute the combinatorial object of standard pairs $\mathcal{S}$ and the initial ideal with respect to $w$.

2. Let $w \in \mathbb{R}^{n}$ be a generic weight vector. Compute the initial ideal $\operatorname{in}_{w}\left(I_{\mathrm{A}}\right)$ with respect to $w$ and obtain its standard pairs $\mathcal{S}\left(\operatorname{in}_{w}\left(I_{A}\right)\right)$. Standard pairs are combinatorial objects which tells us the types of solutions.

3. Use the standard pairs to construct the indicial ideal

$$
\operatorname{ind}_{w}\left(I_{\mathrm{A}}\right)=\bigcap_{\left(\partial^{a}, F\right) \in \mathcal{S}\left(\operatorname{in}_{w}\left(I_{\mathrm{A}}\right)\right)}\left\langle\left(\theta_{j}-a_{j}\right), j \notin F\right\rangle \subset \mathbb{C}\left[\theta_{1}, \theta_{2}, \ldots, \theta_{n}\right],
$$

where $\theta_{i}=c_{i} \partial_{i}$.

4. Write the ideal $\left\langle\mathrm{A} \theta-\kappa^{T}\right\rangle \subset \mathbb{C}\left[\theta_{1}, \theta_{2}, \ldots, \theta_{n}\right]$.

5. The fake indicial ideal with respect to $w$ is given by

$$
\operatorname{fin}_{w}\left(H_{\mathrm{A}}(\kappa)\right):=\operatorname{ind}_{w}\left(I_{\mathrm{A}}\right)+\left\langle A \theta-\kappa^{T}\right\rangle .
$$

6. Compute the roots of $\operatorname{fin}_{w}\left(H_{\mathrm{A}}(\kappa)\right)$. These are called fake exponents and we denote them by $\gamma$.

The canonical series are then given by eq. (2.29). In order to write solutions, we compute the kernel of $\mathrm{A}$ to obtain the generating lattice

$$
\mathcal{L}:=\operatorname{ker}_{\mathbb{Z}} \mathrm{A} .
$$

Finally, we set $[\gamma]_{u_{-}}=0$ whenever $w \cdot u<0$ (see Lemma 3.17 in ref. [45]). Clearly, this property allows us to choose certain weights $w$ that simplify the sums.

We can use Macaulay2 to perform the above operations. An example is provided in appendix B.

\subsection{Examples}

In order to illustrate the methods, we will take two representations of the Gauss hypergeometric function. 


\subsubsection{Double integral Gauss hypergeometric function}

The first part of this example follows [37], where the reader can find details. Suppose we are interested in the following integral

$$
I(\alpha)=\int_{\mathbb{R}_{+}^{2}} \frac{z_{1}^{\alpha_{1}} z_{2}^{\alpha_{2}}}{\left(1+z_{1}+z_{2}+c z_{1} z_{2}\right)^{\beta}} \frac{\mathrm{d} z_{1} \mathrm{~d} z_{2}}{z_{1} z_{2}} .
$$

From the point of view we are adopting, we should think on the more general situation where the polynomial in the denominator has indeterminate coefficients, i.e.,

$$
b(c, z)=c_{1}+c_{2} z_{1}+c_{3} z_{2}+c_{4} z_{1} z_{2} .
$$

Its associated configuration matrix reads

$$
A=\left(\begin{array}{llll}
1 & 1 & 1 & 1 \\
0 & 1 & 0 & 1 \\
0 & 0 & 1 & 1
\end{array}\right)
$$

Then we consider the integral

$$
I_{b}(-\beta,-\alpha)=\int_{\Omega} \frac{z_{1}^{\alpha_{1}} z_{2}^{\alpha_{2}}}{\left(c_{1}+c_{2} z_{1}+c_{3} z_{2}+c_{4} z_{1} z_{2}\right)^{\beta}} \frac{\mathrm{d} z_{1} \mathrm{~d} z_{2}}{z_{1} z_{2}},
$$

where $\Omega$ is a suitable cycle of integration. Following [37] the Newton polytope of $b(c, z)$ can be represented by the inequalities (see eq. (2.7))

$$
\Delta_{b}=\left\{\sigma_{1} \geq 0\right\} \cap\left\{\sigma_{2} \geq 0\right\} \cap\left\{-\sigma_{1} \geq-1\right\} \cap\left\{-\sigma_{2} \geq-1\right\},
$$

and hence, we can read off the vectors $\mu_{i}$ and numbers $\nu_{i}$

$$
\begin{array}{llll}
\mu_{1}=(1,0), & \mu_{2}=(0,1), & \mu_{3}=(-1,0), & \mu_{4}=(0,-1), \\
\nu_{1}=0, & \nu_{2}=0, & \nu_{3}=-1, & \nu_{4}=-1 .
\end{array}
$$

Integration cycles can be obtained taking $\theta=\left(\arg \left(c_{1} / c_{2}\right), \arg \left(c_{1} / c_{3}\right)\right)$. The BFP Theorem states that

$$
I_{b}(-\beta,-\alpha)=\Phi_{b}^{\Theta}(\alpha, \beta, c) \Gamma\left(\alpha_{1}\right) \Gamma\left(\alpha_{2}\right) \Gamma\left(\beta-\alpha_{1}\right) \Gamma\left(\beta-\alpha_{2}\right),
$$

where $\Theta \in \mathbb{T}^{2} \backslash \overline{\mathcal{A}_{b}^{\prime}}$. Taking the representative $\theta \in \Theta$, the entire function reads

$$
\Phi_{b}^{\Theta}(\alpha, \beta, c)=\frac{c_{1}^{\alpha_{1}+\alpha_{2}-\beta} c_{2}^{-\alpha_{2}} c_{3}^{-\alpha_{3}}}{\Gamma(\beta)^{2}}{ }_{2} F_{1}\left(\alpha_{1}, \alpha_{2}, \beta ; 1-\frac{c_{1} c_{4}}{c_{2} c_{3}}\right) .
$$

Taking the point $c^{\prime}=(1,1,1, c)$, we have the coamoeba of $b(z)$ shown in figure 3 , where we see that $(0,0) \notin \overline{\mathcal{A}_{b}^{\prime}}$. There is one connected component of $\mathbb{R}^{2} \backslash \overline{\mathcal{A}_{b}^{\prime}}$ at this point, therefore we have a single solution of $H_{\mathrm{A}}(-\beta,-\alpha)$. Notice that at $c^{\prime}$, we have $\Omega=\operatorname{Arg}^{-1} \theta=$ $\operatorname{Arg}^{-1}\left(\arg \left(c_{1} / c_{2}\right), \arg \left(c_{1} / c_{3}\right)\right)=\operatorname{Arg}^{-1}(0,0)=(0, \infty)^{2}$. Hence, at $c^{\prime}$ we have

$$
I(\alpha)=\frac{\Gamma\left(\alpha_{1}\right) \Gamma\left(\alpha_{2}\right) \Gamma\left(\beta-\alpha_{1}\right) \Gamma\left(\beta-\alpha_{2}\right)}{\Gamma(\beta)^{2}}{ }_{2} F_{1}\left(\alpha_{1}, \alpha_{2}, \beta ; 1-c\right) .
$$




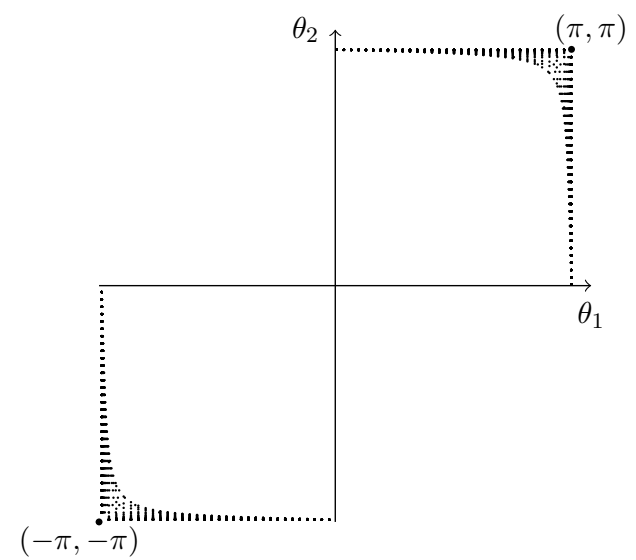

Figure 3. A connected component of the coamoeba(shaded) of $b(z)=1+z_{1}+z_{2}+c z_{1} z_{2}$ drawn in the fundamental domain $[-\pi, \pi] \times[-\pi, \pi]$ of $\mathbb{T}^{2}$ in $\mathbb{R}^{2}$. The coefficient $c$ is near 1 .

It is instructive to recover this result from the canonical series algorithm. Let $w=$ $(0,1,1,1),{ }^{16}$ and $\kappa=\left(-\beta,-\alpha_{1},-\alpha_{2}\right)$. The toric ideal associated with $\mathrm{A}$ reads

$$
I_{\mathrm{A}}=\left\langle\partial_{2} \partial_{3}-\partial_{1} \partial_{4}\right\rangle
$$

We have $\operatorname{in}_{w}\left(I_{\mathrm{A}}\right)=\left\langle\partial_{2} \partial_{3}\right\rangle$, which we use to obtain its standard pairs

$$
\mathcal{S}\left(\operatorname{in}_{w}\left(I_{\mathrm{A}}\right)\right)=\{(1,\{1,3,4\}),(1,\{1,2,4\})\},
$$

therefore

$$
\operatorname{ind}_{w}\left(I_{A}\right)=\left\langle\theta_{2}\right\rangle \cap\left\langle\theta_{3}\right\rangle \text {. }
$$

Thus, we obtain

$$
\operatorname{fin}_{w}\left(H_{\mathrm{A}}(\kappa)\right)=\left\langle\theta_{2} \theta_{3}, \theta_{1}+\theta_{2}+\theta_{3}+\theta_{4}+\beta, \theta_{2}+\theta_{4}+\alpha_{1}, \theta_{3}+\theta_{4}+\alpha_{2}\right\rangle .
$$

Computing its roots leads to

$$
\left\{\gamma_{i}\right\}=\left\{\left(\alpha_{1}-\beta, \alpha_{2}-\alpha_{1}, 0,-\alpha_{2}\right),\left(\alpha_{2}-\beta, 0, \alpha_{1}-\alpha_{2},-\alpha_{1}\right)\right\} .
$$

In addition, computing $\operatorname{ker}(\mathrm{A})$ we have $\mathcal{L}=\mathbb{Z}(1,-1,-1,1)$. Let $u=n(1,-1,-1,1)$, then $u . w=-n$, which means that $[\gamma]_{u_{-}}=0$ for $n>1$. In order to start the sum from $n=0$, we set $u=n(-1,1,1,-1)$ such that $[\gamma]_{u_{-}}=0$ for $n<0$. We then write

$$
u=u_{+}-u_{-}=(0, n, n, 0)-(n, 0,0, n) .
$$

Let us work out the case of $\gamma_{1}$. Eq. (2.29) gives

$$
\phi_{1}=\sum_{n=0}^{\infty} \frac{\left[\gamma_{1}\right]_{u_{-}}}{\left[\gamma_{1}+u\right]_{u_{+}}}\left(c^{u+\gamma_{1}}\right)=c^{\gamma_{1}} \sum_{n=0}^{\infty} \frac{\left[\left(\alpha_{1}-\beta, \alpha_{2}-\alpha_{1}, 0,-\alpha_{2}\right)\right]_{u_{-}}}{\left[\left(\alpha_{1}-\beta, \alpha_{2}-\alpha_{1}, 0,-\alpha_{2}\right)+n(-1,1,1,-1)\right]_{u_{+}}}\left(c^{u}\right),
$$

\footnotetext{
${ }^{16}$ Choices of $w$ are tied with the property that for $w \cdot u<0$ we have $[\gamma]_{u_{-}}=0$. See Algorithm in section 2.4.1.
} 
hence

$$
\phi_{1}=c^{\gamma_{1}} \sum_{n=0}^{\infty} \frac{\left(\beta-\alpha_{1}\right)_{n}\left(\alpha_{2}\right)_{n}}{\left(\alpha_{2}-\alpha_{1}+1\right)_{n}(1)_{n}}\left(\frac{c_{2} c_{3}}{c_{1} c_{4}}\right)^{n}=c^{\gamma_{1}}{ }_{2} F_{1}\left(\beta-\alpha_{1}, \alpha_{2} ; \alpha_{2}-\alpha_{1}+1 ;\left(c_{2} c_{3}\right) /\left(c_{1} c_{4}\right)\right),
$$

where we have used the series representation of the Gauss hypergeometric function (A.3). Similarly, for $\gamma_{2}$ we have

$$
\phi_{2}=c^{\gamma_{2}} \sum_{n=0}^{\infty} \frac{\left(\beta-\alpha_{2}\right)_{n}\left(\alpha_{1}\right)_{n}}{\left(\alpha_{1}-\alpha_{2}+1\right)_{n}(1)_{n}}\left(\frac{c_{2} c_{3}}{c_{1} c_{4}}\right)^{n}=c^{\gamma_{2}}{ }_{2} F_{1}\left(\beta-\alpha_{2}, \alpha_{1} ; \alpha_{1}-\alpha_{2}+1 ;\left(c_{2} c_{3}\right) /\left(c_{1} c_{4}\right)\right) .
$$

Therefore, the solution as linear combination of canonical series reads

$$
\begin{aligned}
I_{b}(-\beta,-\alpha)= & K_{1} c^{\gamma_{1}}{ }_{2} F_{1}\left(\beta-\alpha_{1}, \alpha_{2} ; \alpha_{2}-\alpha_{1}+1 ;\left(c_{2} c_{3}\right) /\left(c_{1} c_{4}\right)\right) \\
& +K_{2} c^{\gamma_{2}}{ }_{2} F_{1}\left(\beta-\alpha_{2}, \alpha_{1} ; \alpha_{1}-\alpha_{2}+1 ;\left(c_{2} c_{3}\right) /\left(c_{1} c_{4}\right)\right) .
\end{aligned}
$$

We proceed to compute the integration constants. We take the noncompact cycle $\Omega=\mathbb{R}_{+}^{2}$. From the roots, we observe that if $c_{3}=0$, then $\phi_{2}$ vanishes and if $c_{2}=0, \phi_{1}$ vanishes. Hence we make

$$
\int_{\mathbb{R}_{+}^{2}} \frac{z_{1}^{\alpha_{1}} z_{2}^{\alpha_{2}}}{\left(c_{1}+c_{2} z_{1}+c_{4} z_{1} z_{2}\right)} \frac{\mathrm{d} z_{1} \mathrm{~d} z_{2}}{z_{1} z_{2}}=\left.\left(K_{1} \phi_{1}+K_{2} \phi_{2}\right)\right|_{c_{3} \rightarrow 0}
$$

which leads to

$$
K_{1}=\frac{\Gamma\left(\alpha_{2}\right) \Gamma\left(\alpha_{1}-\alpha_{2}\right) \Gamma\left(\beta-\alpha_{1}\right)}{\Gamma(\beta)} .
$$

Similarly, we obtain

$$
K_{2}=\frac{\Gamma\left(\alpha_{1}\right) \Gamma\left(\alpha_{2}-\alpha_{1}\right) \Gamma\left(\beta-\alpha_{2}\right)}{\Gamma(\beta)} .
$$

Let us now recover the original integrals by setting $c_{1}=c_{2}=c_{3}=1, c_{4}=c$ in eq. (2.58). We have

$$
\begin{aligned}
I(\alpha)= & c^{-\alpha_{2}} \frac{\Gamma\left(\alpha_{2}\right) \Gamma\left(\alpha_{1}-\alpha_{2}\right) \Gamma\left(\beta-\alpha_{1}\right)}{\Gamma(\beta)}{ }_{2} F_{1}\left(\beta-\alpha_{1}, \alpha_{2} ; \alpha_{2}-\alpha_{1}+1 ; 1 / c\right) \\
& +c^{-\alpha_{1}} \frac{\Gamma\left(\alpha_{1}\right) \Gamma\left(\alpha_{2}-\alpha_{1}\right) \Gamma\left(\beta-\alpha_{2}\right)}{\Gamma(\beta)}{ }_{2} F_{1}\left(\beta-\alpha_{2}, \alpha_{1} ; \alpha_{1}-\alpha_{2}+1 ; 1 / c\right),
\end{aligned}
$$

which equals eq. (2.46) after using the identity (A.9) with $z=1-c$.

\subsubsection{Single integral Gauss hypergeometric function}

Let us now study the univariate integral of the Gauss hypergeometric function. We have

$$
I\left(-\beta_{1},-\beta_{2},-\alpha\right)=\int_{\mathbb{R}_{+}} \frac{z^{\alpha}}{(1+z)^{\beta_{1}}(1+c z)^{\beta_{2}}} \frac{\mathrm{d} z}{z} .
$$


In order to evaluate the integral we consider the (toric) polynomial

$$
b(z)^{\beta}=\left(c_{1}+c_{2} z\right)^{\beta_{1}}\left(c_{3}+c_{4} z\right)^{\beta_{2}} \Longleftrightarrow \mathrm{A}=\left(\begin{array}{llll}
1 & 1 & 0 & 0 \\
0 & 0 & 1 & 1 \\
0 & 1 & 0 & 1
\end{array}\right)
$$

and its associated GKZ system. The integral under consideration reads

$$
I_{b}(\kappa)=\int_{\Omega} \frac{z^{\alpha}}{\left(c_{1}+c_{2} z\right)^{\beta_{1}}\left(c_{3}+c_{4} z\right)^{\beta_{2}}} \frac{\mathrm{d} z}{z},
$$

where $\kappa=\left(-\beta_{1},-\beta_{2},-\alpha\right)$. We have $\mathcal{L}=\mathbb{Z}(1,-1,-1,1)$. Taking the weight vector $w=(0,1,1,1)$, we obtain

$$
\begin{aligned}
\operatorname{fin}_{w}\left(H_{\mathrm{A}}(\kappa)\right) & =\left\langle\theta_{2} \theta_{3}, \beta_{1}+\theta_{1}+\theta_{2}, \beta_{2}+\theta_{3}+\theta_{4}, \alpha+\theta_{2}+\theta_{4}\right\rangle, \\
\left\{\gamma_{i}\right\} & =\left\{\left(-\beta_{1}, 0, \alpha-\beta_{2},-\alpha\right),\left(\alpha-\beta_{1}-\beta_{2}, \beta_{2}-\alpha, 0,-\beta_{2}\right)\right\} .
\end{aligned}
$$

Hence, we have the two series solutions

$$
\begin{aligned}
& \phi_{1}=c^{\gamma_{1}}{ }_{2} F_{1}\left(\beta_{1}, \alpha ; \alpha-\beta_{2}+1 ; \frac{c_{2} c_{3}}{c_{1} c_{4}}\right), \\
& \phi_{2}=c^{\gamma_{2}}{ }_{2} F_{1}\left(\beta_{1}+\beta_{2}-\alpha, \beta_{2} ; \beta_{2}-\alpha+1 ; \frac{c_{2} c_{3}}{c_{1} c_{4}}\right) .
\end{aligned}
$$

Specializing to our case, we have $c_{1}=c_{2}=c_{3}=1$ and $c_{4}=c$, therefore the solution of the integral is given by

$$
\begin{aligned}
I\left(-\beta_{1},-\beta_{2},-\alpha\right)= & K_{1} c^{-\alpha}{ }_{2} F_{1}\left(\beta_{1}, \alpha ; \alpha-\beta_{2}+1 ; 1 / c\right) \\
& +K_{2} c^{-\beta_{2}}{ }_{2} F_{1}\left(\beta_{1}+\beta_{2}-\alpha, \beta_{2} ; \beta_{2}-\alpha+1 ; 1 / c\right),
\end{aligned}
$$

where the constants can be obtained by setting $c_{2}$ and $c_{3}$ to zero in eq. (2.65) choosing $\Omega=\mathbb{R}_{+}$. The final result reads

$$
\begin{aligned}
I\left(-\beta_{1},-\beta_{2},-\alpha\right)= & \frac{\Gamma(\alpha) \Gamma\left(\beta_{2}-\alpha\right)}{\Gamma\left(\beta_{2}\right)} c^{-\alpha}{ }_{2} F_{1}\left(\beta_{1}, \alpha ; \alpha-\beta_{2}+1 ; 1 / c\right) \\
& +c^{-\beta_{2}} \frac{\Gamma\left(\alpha-\beta_{2}\right) \Gamma\left(\beta_{1}+\beta_{2}-\alpha\right)}{\Gamma\left(\beta_{1}\right)}{ }_{2} F_{1}\left(\beta_{1}+\beta_{2}-\alpha, \beta_{2} ; \beta_{2}-\alpha+1 ; 1 / c\right),
\end{aligned}
$$

which evaluates to (2.63) after using eq. (A.9) with $z=1-c$.

\section{Feynman integrals as A-hypergeometric functions}

In this section we will interpret Feynman integrals as particular points of A-hypergeometric functions. First, we will introduce the parametric representation based on $g=\mathcal{U}+\mathcal{F}$ used in ref. [44] by Lee and Pomeransky. Later, we will give our proposal for defining GKZ systems based on $g$. Examples will be presented at the end of this section. 


\subsection{Lee-Pomeransky representation of Feynman integrals}

Let us consider a typical Feynman integral in Euclidean space in dimensional regularization. A $L$-loop integral with $N$ propagators and $E$ independent external momenta may be written as follows

$$
I_{F}(\alpha)=\int_{\mathbb{R}^{L}}\left(\prod_{i=1}^{L} \frac{\mathrm{d}^{d} k_{i}}{\pi^{d / 2}}\right) \frac{1}{D_{1}^{\alpha_{1}} \cdots D_{N}^{\alpha_{N}}}
$$

where the inverse propagators are of the form

$$
D_{i}=\left(M_{i}\right)^{r s} k_{r} \cdot k_{s}+2\left(Q_{i}\right)^{r s} k_{r} \cdot p_{s}+J_{i} .
$$

The matrices, $M_{i}, Q_{i}$, and $J_{i}$ have dimensions $L \times L, L \times E$, and $1 \times 1$, respectively. Integration over loop momenta through Feynman parameters and a Mellin transform leads to the Lee-Pomeransky parametric representation [44]

$$
I_{F}(\alpha)=\xi_{\Gamma_{\alpha}} \int_{\mathbb{R}_{+}^{N}}\left(\prod_{i=1}^{N} \frac{\mathrm{d} z_{i}}{z_{i}} z_{i}^{\alpha_{i}}\right) \frac{1}{g(z)^{d / 2}}=\xi_{\Gamma_{\alpha}} \int_{\mathbb{R}_{+}^{N}} \frac{z^{\alpha}}{g(z)^{d / 2}} \mathrm{~d} \eta_{N}
$$

where we have used the multi-index notation in the second equality and $\mathbb{R}_{+}=(0, \infty)$. The overall factor and the polynomial $g(z)$ are defined as follows:

$$
\begin{aligned}
\xi_{\Gamma_{\alpha}} & :=\frac{\Gamma(d / 2)}{\Gamma\left((L+1) d / 2-\sum_{i=1}^{N} \alpha_{i}\right) \prod_{i=1}^{N} \Gamma\left(\alpha_{i}\right)}, \\
g(z) & :=\mathcal{U}+\mathcal{F}
\end{aligned}
$$

where $\mathcal{U}$ and $\mathcal{F}$ are the Symanzik polynomials. In order to compute them, we construct the matrices

$$
M^{r s}=\sum_{i=1}^{N} z_{i} M_{i}^{r s}, \quad Q^{r}=\sum_{i=1}^{N} z_{i} Q_{i}^{r s} p_{s}, \quad J=\sum_{i=1}^{N} z_{i} J_{i} .
$$

We then have

$$
\mathcal{U}=\operatorname{det}(M), \quad \mathcal{F}=\operatorname{det}(M)\left(J-\left(M^{-1}\right)^{i j} Q^{i} \cdot Q^{j}\right),
$$

where $\mathcal{F}$ is appropriately scaled in order to make it dimensionless. The polynomial $\mathcal{U}$ is a homogeneous polynomial of degree $L$ and the polynomial $\mathcal{F}$ is homogeneous of degree $L+1$. Hence $g(z)$ is an inhomogeneous polynomial of degree $L+1$. In Euclidean kinematics, the Symanzik polynomials $\mathcal{U}, \mathcal{F}$ are positive semi-definite functions of the Feynman parameters. These polynomials can also be obtained from the topology of the graphs and their properties are summarized in refs. [58-60]. 


\subsection{Feynman integrals and canonical series}

A suitable definition for computational algebra purposes would be to consider Feynman integrals as a linear combinations of their canonical series, whenever we can compute them. As they stand, Feynman integrals will not satisfy the system of PDEs associated with a GKZ system (eqs. (2.13)-(2.14)) because the polynomial $g(z)$ has fixed coefficients.

Accordingly, the first step in our construction will be to take $g(z)$ and consider its associated toric polynomial $g(c, z)$. We will demand that this polynomial furnish GKZ system in the sense of eq. (2.29). In particular, this means that $g(c, z)$ must lead to matrices of $\operatorname{co}(\mathrm{A})>0$ in order to compute a generating lattice and follow the canonical series algorithm. Since we have a single polynomial, the codimension of the matrix A associated with $g(c, z)$ is given by $\operatorname{co}(\mathrm{A})=n-N-1$, where $n$ is the number of monomial terms in $g(c, z)$ with common exponent vectors. Therefore, we will have $\operatorname{co}(\mathrm{A})=0$ when the number of terms in $g(c, z)$ equals the number of rows in $\mathrm{A}$, or equivalently when $n=N+1$. A typical example of this situation is the polynomial of the $L$-loop massless cantaloupe graph (figure 6). For instance, let us consider the case $L=1$, i.e., the massless bubble graph (figure 4). The $g(z)$ polynomial of this graph is given by $g(z)=z_{1}+z_{2}+s z_{1} z_{2}$, therefore

$$
g^{\text {bubble }}(c, z)=c_{2} z_{1}+c_{3} z_{2}+c_{4} z_{1} z_{2} \Longleftrightarrow \mathrm{A}^{\text {bubble }}=\left(\begin{array}{lll}
1 & 1 & 1 \\
1 & 0 & 1 \\
0 & 1 & 1
\end{array}\right) .
$$

The codimension of $A^{\text {bubble }}$ is zero and hence ker $A=\emptyset$. Therefore, we cannot use this matrix to represent a solution of a GKZ system as canonical series in the sense of eq. (2.29). However, in this simple example we know that the result of the massless bubble integral can be recovered from the one-mass bubble integral by taking the limit of the mass going to zero. Furthermore, as we will see in the examples, the matrix associated with the one-mass bubble integral is given by

$$
A^{\text {one-mass }}=\left(\begin{array}{llll}
1 & 1 & 1 & 1 \\
1 & 0 & 1 & 0 \\
0 & 1 & 1 & 2
\end{array}\right)
$$

which has $\operatorname{co}\left(A^{\text {one-mass }}\right)=1$. Since the one-mass bubble evaluates to a Gauss hypergeometric function, we may consider the massless bubble as a special case of a ${ }_{2} F_{1}\left(a, b, c ; p^{2} / m^{2}\right)$ function, for some $a, b, c$ depending on the powers of the propagators and the dimension. An equivalent alternative provided by GKZ systems is to deform the polynomial by adding an arbitrary constant $r(z)=c_{1}$ to $g^{\text {bubble }}(c, z)$ and consider instead

$$
g_{r}(c, z)=c_{1}+c_{2} z_{1}+c_{4} z_{2}+c_{4} z_{1} z_{2} \Longleftrightarrow \mathrm{A}^{r}=\left(\begin{array}{llll}
1 & 1 & 1 & 1 \\
0 & 1 & 0 & 1 \\
0 & 0 & 1 & 1
\end{array}\right),
$$

where the constant $c_{1}$ can be set to zero at the end of the computation. This matrix corresponds to the GKZ system of a Gauss hypergeometric function. We will work out explicitly this example in section 3.3.1. There, we will take the limit $c_{1} \rightarrow 0$ of a Gauss 
hypergeometric function as we would do for the mass in the case of a one-mass bubble. The choice of a deformation can be made systematically as we will see for the $L$-loop cantaloupe graph.

Therefore, in cases where $g(c, z)$ leads to a configuration matrix of $\operatorname{co}(\mathrm{A})=0$, we will consider a deformation of $g(c, z)$ demanding that it defines a solution of a GKZ system in the sense of eq. (2.29), thus allowing us to construct canonical series solutions. Recall that $\operatorname{deg}(\mathcal{U})=L$ and $\operatorname{deg}(\mathcal{F})=L+1$. We may choose any polynomial $r(z)$ of $\operatorname{deg}(r)<L$ and define

$$
g_{r}(c, z):=r(c, z)+\mathcal{U}(c)+\mathcal{F}(c)
$$

with the requirement that the associated matrix has $\operatorname{co}(\mathrm{A})>0$. Here $r(c, z)$ denotes the toric polynomial associated with $r(z)$. Similarly, $\mathcal{U}(c)$ and $\mathcal{F}(c)$ denote the Symanzik polynomials where the coefficients now are considered as variables. We have checked for the integral of a massless $L$-loop cantaloupe graph, up to 5-loop, that this definition leads to well behaved GKZ systems and that we can recover the original integral using canonical series.

The second step in our construction will be to consider Euler-type solutions and series solutions of the GKZ systems furnished with $g_{r}(c, z)$. Let us first define the class of Euler integrals associated with the Feynman integral (3.3). We will drop the overall factor $\xi_{\Gamma_{\alpha}}$, which is a nonzero constant independent of the kinematics and instead consider

$$
I_{F}(\alpha) / \xi_{\Gamma_{\alpha}} \longmapsto I_{g_{r}}(-d / 2,-\alpha):=\int_{\Omega} \frac{z^{\alpha}}{g_{r}(c, z)^{d / 2}} \mathrm{~d} \eta_{N},
$$

which is a toric generalization of eq. (3.3), i.e., where the deformation has been introduced and the coefficients of $g_{r}(z)$ are considered as variables. Notice that at this point, the noncompact cycle $\Omega$ will not be in general $\mathbb{R}_{+}^{N}$ and it will be determined by the coamoeba of $g_{r}(c, z)$ (section 2.3.1).

Let us first show that the class of integrals obtained from $g_{r}(c, z)$ is A-hypergeometric. ${ }^{17}$ We have the following theorem, which is a specialization of theorem 2.7 in ref. [35] for the case of a single polynomial $g_{r}(c, z)$. That this theorem still holds for noncompact cycles is discussed in refs. [38, 39].

Theorem. Let $g_{r}(c, z)$ be the deformed polynomial in $N$ variables obtained from $g(c, z)=$ $\mathcal{U}(c)+\mathcal{F}(c)$, where $\mathcal{F}(c)$ and $\mathcal{U}(c)$ are obtained by considering the coefficients appearing in the Symanzik polynomials as variables. $g_{r}(c, z)$ is obtained by introducing a deformation $r(c, z)$ demanding that its matrix satisfies $c o(\mathrm{~A})>0$. Let $A=\left(\begin{array}{llll}a_{1} & a_{2} \cdots & a_{n}\end{array}\right)$ be the configuration matrix associated with $g_{r}(c, z)$ and consider the polynomial with indeterminate generic coefficients

$$
g_{r}(c, z)=\sum_{i=1}^{n} c_{i} z^{a_{i}}, \quad c_{i} \in \mathbb{C}_{*}
$$

\footnotetext{
${ }^{17} \mathrm{~A}$ related construction — which does not introduce deformations - has been considered in ref. [41] for the special case where the powers of the propagators are given by $\alpha=(1, \ldots, 1)$. Here we consider generic powers in the propagators as dictated by canonical series solutions.
} 
Let $\mathrm{A}$ be its associated $(N+1) \times n$ matrix

$$
A=\left(\begin{array}{cccc}
1 & 1 & \ldots & 1 \\
a_{1} & a_{2} & \ldots & a_{n}
\end{array}\right) .
$$

The Euler-Mellin integral

$$
I_{g_{r}}(\kappa)=\int_{\Omega} \frac{z^{\alpha}}{g_{r}(c, z)^{d / 2}} \mathrm{~d} \eta_{N}
$$

is a solution of the A-hypergeometric system $H_{\mathrm{A}}(\kappa)$ of degree $\kappa=(-d / 2,-\alpha)$. Noncompact cycles $\Omega$ can be obtained by taking the coamoeba of $g_{r}(c, z)$ and choosing representatives $\theta$ of connected components $\Theta \in \mathbb{R}^{N} \backslash{\overline{\mathcal{A}^{\prime}}}_{g_{r}}$ (see section (2.3.1)).

Proof. The proof goes along the lines of ref. [39] specializing to the nonhomogeneous case and the case of a single polynomial. Let us consider first eq. (2.13). We have

$$
\begin{aligned}
\left(\partial_{1}\right)^{u_{1}}\left(\partial_{2}\right)^{u_{2}} \cdots & \left(\partial_{n}\right)^{u_{n}} I_{g_{r}}(\kappa)=(-d / 2)\left(\partial_{1}\right)^{u_{1}}\left(\partial_{2}\right)^{u_{2}} \cdots\left(\partial_{n}\right)^{u_{n}-1} \int_{\Omega} \mathrm{d} \eta_{N} z^{\alpha} g_{r}(c, z)^{-d / 2-1} z^{a_{i}} \\
& =(-d / 2)_{\left(\left|u_{n}\right|\right)}\left(\partial_{1}\right)^{u_{1}}\left(\partial_{2}\right)^{u_{2}} \cdots\left(\partial_{n-1}\right)^{u_{n-1}} \int_{\Omega} \mathrm{d} \eta_{N} z^{\alpha} g_{r}(c, z)^{-d / 2-\left|u_{n}\right|} z^{\left|u_{n}\right| a_{n}} \\
& =(-d / 2)_{\left(\left|u_{1}\right|+\cdots+\left|u_{n}\right|\right)} \int_{\Omega} \mathrm{d} \eta_{N} z^{\alpha} g_{r}(c, z)^{-d / 2-\left|u_{1}\right|-\cdots-\left|u_{n}\right|} z^{\left|u_{1}\right| a_{1}+\cdots+\left|u_{n}\right| a_{n}},
\end{aligned}
$$

where $(\rho)_{(x)}$ denotes the falling factorial. Similarly

$$
\begin{aligned}
\left(\partial_{1}\right)^{v_{1}}\left(\partial_{2}\right)^{v_{2}} & \cdots\left(\partial_{n}\right)^{v_{n}} I_{g_{r}}(\kappa) \\
& =(-d / 2)_{\left(\left|v_{1}\right|+\cdots+\left|v_{n}\right|\right)} \int_{\Omega} \mathrm{d} \eta_{N} z^{\alpha} g_{r}(c, z)^{-d / 2-\left|v_{1}\right|-\cdots-\left|v_{n}\right|} z^{\left|v_{1}\right| a_{1}+\cdots+\left|v_{n}\right| a_{n}},
\end{aligned}
$$

and from $\mathrm{A} u=\mathrm{A} v$, the result $\left(\partial^{u}-\partial^{v}\right) I_{g_{r}}(\kappa)=0$ follows.

Let us now focus on the second set of differential equations (2.14). Consider the first row of $\mathrm{A}$ in eq. (3.14). We have

$$
\begin{aligned}
\left(c_{1} \partial_{1}+c_{2} \partial_{2}+\cdots+c_{n} \partial_{n}\right) I_{g_{r}}(\kappa) & =\int_{\Omega} \mathrm{d} \eta_{N} z^{\alpha}(-d / 2) g_{r}(c, z)^{-d / 2-1}\left(c_{1} z^{a_{1}}+\cdots+c_{n} z^{a_{n}}\right) \\
& =(-d / 2) I_{g_{r}}(\kappa) .
\end{aligned}
$$

Similarly, for $i>1$

$$
\begin{aligned}
\sum_{j=1}^{n} a_{i j} c_{j} \partial_{j} I_{g_{r}}(\kappa) & =\sum_{j=1}^{n} a_{i j} \int_{\Omega} \mathrm{d} \eta_{N} z^{\alpha}(-d / 2) g_{r}(c, z)^{-d / 2-1}\left(c_{j} z^{a_{j}}\right) \\
& =\int_{\Omega} \mathrm{d} \eta_{N} z^{\alpha}(-d / 2) g_{r}(c, z)^{-d / 2-1}\left(z_{i} \frac{\partial}{\partial z_{i}} g_{r}(c, z)\right) \\
& =\int_{\Omega} \mathrm{d} \eta_{N} z^{\alpha}\left(z_{i} \frac{\partial}{z_{i}} g_{r}(c, z)^{-d / 2}\right) \\
& =-\alpha_{i} I_{g_{r}}(\kappa),
\end{aligned}
$$

where we have used integration by parts in the last equality. This completes the proof. 
In this way, we have generated a class of integrals related with the Lee-Pomeransky representation of Feynman integrals. Feynman integrals will correspond to special cases (points) of A-hypergeometric functions whenever the cycle $\Omega$ can be taken as $\mathbb{R}_{+}^{N}$ and the coefficients $c$ can be taken as functions of the kinematic invariants. The behavior of the integral as the coefficients $c$ vary can be studied through eq. (2.22). This representation is also useful as it provides noncompact cycles at the cost of imposing conditions on $\kappa$. However, this can be sorted out by analytic continuation and it can be shown that, as a function of the variables $c$, eq. (2.22) is A-hypergeometric everywhere. Here $c$ is taken in $\mathbb{C}^{n} \backslash \Sigma_{\mathrm{A}}$, where $\Sigma_{\mathrm{A}}$ denotes the singular locus of all A-hypergeometric functions (Theorem 4.2 in [38]). The main difficulty in this approach is to choose a representative of some connected component $\Theta$ in $\mathbb{R}^{N} \backslash \overline{\mathcal{A}_{g_{r}}^{\prime}}$. In other words, we have to select a point such that the cycle is nonvanishing on the set $\operatorname{Arg}^{-1}(\theta)$, where $\theta \in \Theta$. The integration region of the Feynman integral, namely $\mathbb{R}_{+}^{N}$, suggest taking any $\theta=\left(\arg \left(f_{1}(c)\right), \ldots, \arg \left(f_{N}(c)\right)\right), f_{i}(c)>0$ provided $\theta \notin \overline{\mathcal{A}_{g_{r}}^{\prime}}$ (see e.g. figure 3). This gives one connected component of $\operatorname{vol}(\mathrm{A})$ many ones and a possible integration cycle. ${ }^{18}$

On the other hand, logarithm-free canonical series allows us to study the behavior of the solution space under variations of the parameters $\kappa$ at nonsingular points [57,61]. More important, once we have identified the above integrals as solutions of a GKZ system, we can use the statement in eq. (2.32) relating Euler-type integrals and canonical series. For our case, this statement reads

$$
\int_{\Omega} \frac{z^{\alpha}}{g_{r}(c, z)^{d / 2}} \mathrm{~d} \eta_{N}=K_{1} \phi_{1}+\cdots+K_{M} \phi_{M}
$$

where $\phi_{1}, \ldots, \phi_{M}$ are the canonical series associated with A. Fake exponents $\gamma$ for each $\phi_{1}, \ldots, \phi_{M}$ can be obtained from the SST algorithm in section 2.4.1. This equality is fundamental for our purposes as it allows both to take the limit of the deformation to zero and computing the integrations constants. Let us discuss these limits.

The form of the series solution for some fake exponent $\gamma$ reads

$$
\phi_{\gamma}=c^{\gamma} \sum_{u \in \mathcal{L}} \frac{[\gamma]_{u_{-}}}{[\gamma+u]_{u_{+}}} c^{u} .
$$

These are characterized by a weight vector $w \in \mathbb{R}^{n}$, which selects a common domain of convergence $\mathcal{U}_{w}$ (see Theorem 2.5.16 in [40]). In addition, we have the restriction $[\gamma]_{u_{-}}=0$ for $u . w<0$. Taking the limit of the deformation to zero amounts to take some of the coefficients in $c=\left(c_{1}, \ldots, c_{n}\right)$ to zero in eq. (3.17) and similarly for the remaining canonical series. On the l.h.s. of eq. (3.16), taking this limit amounts to recover the undeformed integral we started with. Let us clarify this point. The integral in the l.h.s. of (3.16) is a well defined A-hypergeometric function provided $c$ are generic and the cycle is taken in some $\theta \notin \overline{\mathcal{A}_{g_{r}}^{\prime}}$. The r.h.s. of (3.16) is an asymptotic expansion of such integral. Therefore, the limits on the l.h.s. correspond to special values of the A-hypergeometric functions in the r.h.s.. A judicious choice of the deformation ensures that this limit can be taken systematically as we will see in the examples.

\footnotetext{
${ }^{18}$ For all real positive coefficients in $g(z)$, i.e., the nontoric polynomial, we can ensure that 0 is in $\mathbb{T}^{N}[38]$.
} 
In general, this limit will not be smooth as it will require the evaluation of Ahypergeometric functions on their singular points ${ }^{19}$ and therefore we may require analytic continuation of the corresponding A-hypergeometric function. This can be seen as follows. A generic canonical series solutions can be understood as a function in $\operatorname{co}(A)$ variables. Setting one of these variables to zero may require a transformation of a variable to, say, its inverse and hence we require analytic continuation. A judicious choice of the weight vector $w$ can simplify taking this limit, since it provides the condition $[\gamma]_{u_{-}}=0$ for $u . w<0$, thus determining the arrangement of the powers of $c^{u}$ in eq. (3.17). As we will see for the $L$-loop cantaloupe graph, we can systematically choose a deformation and a weight vector to take this limit. However, we will leave as a conjecture that this this limit can always be taken.

For integration constants, we will use the information available on the fake exponents following ref. [40]. The initial series in eq. (3.17) are given by $c^{\gamma}$. Therefore, the positions of the 0 's on each $\gamma$ will tell us which elements in $c$ we have to set to zero in order to compute the integration constants.

Let us give some final comments. Notice that in order to recover the original Feynman integral - in agreement with our definition of the polynomials associated with the GKZ system and of the integral (3.15) — we must set coefficients $c$ to their kinematic values at the end of the computation. The integral (3.15) can be understood as a holomorphic function on $\mathcal{U}_{w}$. This observation gives us a nice way of deducing the long known fact that convergent Feynman integrals are functions of a Nilsson class [2], which is clear from their canonical series.

We end with our prescription to compute integration constants.

Integration constants. Suppose that we obtain $M$ fake exponents from the SST algorithm. Let us isolate an exponent, say, $\gamma=\left(\gamma^{1}, \ldots, \gamma^{n}\right)$ and suppose it contains $k<n$ zeros in positions $\sigma_{1}, \ldots, \sigma_{k}$. Take the coefficients associated with those positions to zero and consider

$$
\begin{gathered}
\left.I_{g_{r}}(\kappa)\right|_{c_{\sigma_{1}} \rightarrow 0}=\left(K_{1} \phi_{1}+\cdots+K_{M} \phi_{M}\right)||_{\sigma_{\sigma_{1}} \rightarrow 0}, \\
\vdots \\
c_{\sigma_{k}} \rightarrow 0
\end{gathered}
$$

where we take $\Omega=\mathbb{R}_{+}^{N}$ as the cycle in the l.h.s. . This procedure will compute a single coefficient. We repeat the process until we have computed all of them.

\subsection{Examples of $\operatorname{co}(A)=0$}

The purpose of $\operatorname{co}(A)=0$ examples is to show how to deal with the appearance of a deformation.

Structure of the examples. In the following examples we will omit the overall gamma factors $\xi_{\Gamma_{\alpha}}$ and hence consider

$$
I(\alpha):=I_{F}(\alpha) / \xi_{\Gamma_{\alpha}}
$$

\footnotetext{
${ }^{19}$ See ref. [37] and the example in section 3.3.1.
} 


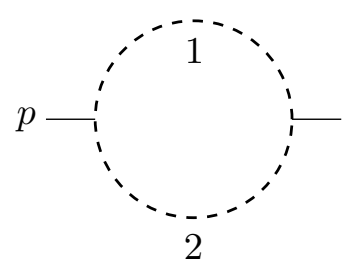

Figure 4. Bubble graph: $s=-p^{2}$.

along with its toric version $I_{g_{r}}(\kappa)$. The vector $\kappa$ has the form

$$
\kappa=\left(-d / 2,-\alpha_{1}, \ldots,-\alpha_{N}\right),
$$

where $N$ is the number of propagators. We assume that the powers of the propagators have generic noninteger complex values. This simplifies the discussion since the sum runs over $\mathcal{L}=\operatorname{ker}_{\mathbb{Z}} \mathrm{A}$. The kernel of $\mathrm{A}$ leads to a rank $\operatorname{co}(\mathrm{A})$ lattice. For a single generator, we write

$$
\mathcal{L}:=\mathbb{Z}\left(a_{1}, \ldots, a_{n}\right), \quad u=n\left(a_{1}, \ldots, a_{n}\right), \quad a_{i} \in \mathbb{Z} .
$$

We set

$$
\beta=d / 2 .
$$

In order to indicate the $i$-th component of a root vector $\gamma_{r}$ we write $\gamma_{r}^{i}$.

\subsubsection{Massless bubble}

The simplest example is the massless bubble (figure 4) with inverse propagators

$$
D_{1}=(k)^{2}, \quad D_{2}=(k-p)^{2},
$$

where $s=-p^{2}$. We have

$$
g(z)=z_{1}+z_{2}+s z_{1} z_{2}
$$

This polynomial leads to a matrix of codimension $\operatorname{co}(\mathrm{A})=0$, hence we introduce a deformation $r(z)=c_{1}$ and consider instead

$$
g_{r}(c, z)=c_{1}+c_{2} z_{1}+c_{3} z_{2}+c_{4} z_{1} z_{2} \Longleftrightarrow \mathrm{A}=\left(\begin{array}{llll}
1 & 1 & 1 & 1 \\
0 & 1 & 0 & 1 \\
0 & 0 & 1 & 1
\end{array}\right) .
$$

Thus, we have

$$
I_{g_{r}}(\kappa)=\int_{\operatorname{Arg}^{-1} \theta} \frac{z_{1}^{\alpha_{1}} z_{2}^{\alpha_{2}}}{\left(c_{1}+c_{2} z_{1}+c_{3} z_{2}+c_{4} z_{1} z_{2}\right)^{\beta}} \frac{\mathrm{d} z_{1}}{z_{1}} \frac{\mathrm{d} z_{2}}{z_{2}}
$$

for $\theta=\left(\arg \left(c_{1} / c_{3}\right), \arg \left(c_{1} / c_{2}\right)\right)$. We have studied this integral in section 2.5, where we established that

$$
I_{g_{r}}(\kappa)=\frac{\Gamma\left(\alpha_{1}\right) \Gamma\left(\alpha_{2}\right) \Gamma\left(\beta-\alpha_{1}\right) \Gamma\left(\beta-\alpha_{2}\right)}{\Gamma(\beta)^{2}} c_{1}^{\alpha_{1}+\alpha_{2}-\beta} c_{2}^{-\alpha_{1}} c_{3}^{-\alpha_{2}}{ }_{2} F_{1}\left(\alpha_{1}, \alpha_{2} ; \beta ; 1-\frac{c_{1} c_{4}}{c_{2} c_{3}}\right) .
$$




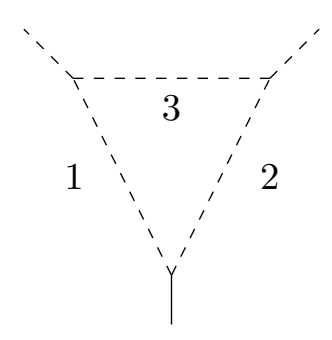

Figure 5. Triangle graph: $s=-\left(p_{1}+p_{2}\right)^{2}$.

In order to recover our Feynman integral we have to take the limit $c_{1} \rightarrow 0$. The limit has to be taken carefully as $c_{1}$ appears both as a factor and in the argument of the hypergeometric function. Using the identity (A.7) we have

$$
{ }_{2} F_{1}\left(\alpha_{1}, \alpha_{2} ; \beta ; 1-\frac{c_{1} c_{4}}{c_{2} c_{3}}\right)=\left(\frac{c_{1} c_{4}}{c_{2} c_{3}}\right)^{\left(\beta-\alpha_{1}-\alpha_{2}\right)}{ }_{2} F_{1}\left(\beta-\alpha_{1}, \beta-\alpha_{2} ; \beta ; 1-\frac{c_{1} c_{4}}{c_{2} c_{3}}\right) .
$$

Therefore

$$
\begin{aligned}
I_{g_{r}}(\kappa)= & \frac{\Gamma\left(\alpha_{1}\right) \Gamma\left(\alpha_{2}\right) \Gamma\left(\beta-\alpha_{1}\right) \Gamma\left(\beta-\alpha_{2}\right)}{\Gamma(\beta)^{2}} \\
& \times c_{2}^{\alpha_{2}-\beta} c_{3}^{\alpha_{1}-\beta} c_{4}^{\beta-\alpha_{1}-\alpha_{2}}{ }_{2} F_{1}\left(\beta-\alpha_{1}, \beta-\alpha_{2} ; \beta ; 1-\frac{c_{1} c_{4}}{c_{2} c_{3}}\right) .
\end{aligned}
$$

Finally, taking the limit $c_{1}=0$, setting $c_{2}=c_{3}=1$, and $c_{4}=s$ we recover the desired result

$$
I(\alpha)=\frac{\Gamma\left(\beta-\alpha_{1}\right) \Gamma\left(\beta-\alpha_{2}\right) \Gamma\left(\alpha_{1}+\alpha_{2}-\beta\right)}{\Gamma(\beta)} s^{\left(\beta-\alpha_{1}-\alpha_{2}\right)},
$$

where we have used identity (A.6). Notice that in order to take this limit, we have evaluated eq. (3.23) on one of the singular points of the Gauss hypergeometric function, thus requiring an Euler transformation.

\subsubsection{The single-scale massless triangle graph}

Let us now turn our attention to those limits through canonical series. Let us consider the triangle graph in figure 5. The inverse propagators read

$$
D_{1}=\left(k_{1}-p_{1}\right)^{2}, \quad D_{2}=\left(k_{1}+p_{2}\right), \quad D_{3}=k_{1}^{2},
$$

where $-p_{1}^{2}=-p_{2}^{2}=0$. Computing the relevant polynomial and taking the deformation $r(z)=c_{1}$ leads to

$$
g_{r}(c, z)=c_{1}+c_{2} z_{1}+c_{3} z_{2}+c_{4} z_{3}+c_{5} z_{1} z_{2} \Longleftrightarrow \mathrm{A}=\left(\begin{array}{ccccc}
1 & 1 & 1 & 1 & 1 \\
0 & 1 & 0 & 0 & 1 \\
0 & 0 & 1 & 0 & 1 \\
0 & 0 & 0 & 1 & 0
\end{array}\right),
$$


where, at the end of the computation, we will make the identifications $c_{2}=c_{3}=c_{4}=1$ and $c_{5}=s=-\left(p_{1}+p_{2}\right)^{2}$. The integral reads

$$
I_{g_{r}}(\kappa)=\int_{\Omega} \mathrm{d} \eta_{3} \frac{z_{1}^{\alpha_{1}} z_{2}^{\alpha_{2}} z_{3}^{\alpha_{3}}}{\left(c_{1}+c_{2} z_{1}+c_{3} z_{2}+c_{4} z_{3}+c_{5} z_{1} z_{2}\right)^{\beta}} .
$$

Computing $\operatorname{ker}(\mathrm{A})$, we have

$$
\mathcal{L}=\mathbb{Z}(1,-1,-1,0,1) \Rightarrow u=n(1,-1,-1,0,1) .
$$

Choosing $w=(1,0,0,0,0)$, we have $u . w=n$, hence $\left[\gamma_{i}\right]_{u_{-}}=0$ for $n<0$. Setting $A=\alpha_{1}+\alpha_{2}+\alpha_{3}$, the fake indicial ideal and its roots read

$$
\begin{aligned}
\operatorname{fin}_{w}\left(H_{\mathrm{A}}(\kappa)\right) & =\left\langle\theta_{1} \theta_{5}, \beta+\theta_{1}+\theta_{2}+\theta_{3}+\theta_{4}+\theta_{5}, \alpha_{1}+\theta_{2}+\theta_{5}, \alpha_{2}+\theta_{3}+\theta_{5}, \alpha_{3}+\theta_{4}\right\rangle, \\
\left\{\gamma_{i}\right\} & =\left\{\left(0, \alpha_{2}+\alpha_{3}-\beta, \alpha_{1}+\alpha_{3}-\beta,-\alpha_{3},-\alpha+\beta\right),\left(A-\beta,-\alpha_{1},-\alpha_{2},-\alpha_{3}, 0\right)\right\} .
\end{aligned}
$$

Inserting the roots in eq. (2.29) gives

$$
\phi_{i}=c^{\gamma_{i}} \sum_{n \geq 0} \frac{\left[\gamma_{i}\right]_{(0, n, n, 0,0)}}{\left[\gamma_{i}+(n,-n,-n, 0, n)\right]_{(n, 0,0,0, n)}}\left(\frac{c_{1} c_{5}}{c_{2} c_{3}}\right)^{n},
$$

which leads to the series

$$
\begin{aligned}
& \phi_{1}=c^{\gamma_{1}} \sum_{n \geq 0} \frac{\left(\beta-\alpha_{2}-\alpha_{3}\right)_{n}\left(\beta-\alpha_{1}-\alpha_{3}\right)_{n}}{(1)_{n}(\beta-A+1)_{n}}\left(\frac{c_{1} c_{5}}{c_{2} c_{3}}\right)^{n}, \\
& \phi_{2}=c^{\gamma_{2}} \sum_{n \geq 0} \frac{\left(\alpha_{1}\right)_{n}\left(\alpha_{2}\right)_{n}}{(-\beta+A+1)_{n}(1)_{n}}\left(\frac{c_{1} c_{5}}{c_{2} c_{3}}\right)^{n} .
\end{aligned}
$$

Integration constants can be easily computed by setting $c_{1}=0$ and $c_{5}=0$ in eq. (3.29) with $\Omega=\mathbb{R}_{+}^{3}$. We write them collectively as

$$
K_{r}=\frac{1}{\Gamma(\beta)} \prod_{i \neq 0} \Gamma\left(-\gamma_{r}^{i}\right)
$$

Taking the limit $c_{1} \rightarrow 0$ and setting $c_{2}=c_{3}=c_{4}=1, c_{5}=s$, we obtain

$$
I(\alpha)=\frac{\Gamma\left(\beta-\alpha_{2}-\alpha_{3}\right) \Gamma\left(\beta-\alpha_{1}-\alpha_{3}\right) \Gamma\left(\alpha_{3}\right) \Gamma(\beta-A)}{\Gamma(\beta)} s^{\beta-A} .
$$

Let us remark that in this example we have set $c_{1}$ to zero in order to compute one of the integration constants - as can be seen from the roots - therefore reaching a tautology. We can fix this by choosing an appropriate weight vector such that none of the roots contain zero in position 1 . This leads to a more complicated version of the canonical series which will require analytic continuation in order to take the limit $c_{1} \rightarrow 0$. Since we want to interpret Feynman integrals with codimension zero matrix as a certain limiting cases of A-hypergeometric functions, we do not worry about this situation. In fact, as we will see in the next example, we can take advantage of this by choosing a weight vector such that all massless cantaloupe graphs are defined by the coefficients of a linear combination of two Gauss hypergeometric functions. 


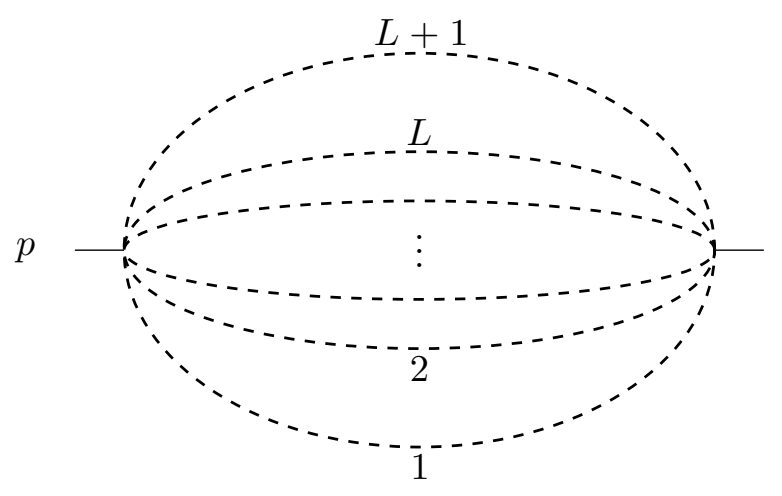

Figure 6. Massless cantaloupe graph.

\subsubsection{The massless $L$-loop cantaloupe graph}

Let us consider the case of the $L$-loop cantaloupe graph (a.k.a. banana graph) shown in figure 6 . We parametrize the inverse propagators as follows

$$
D_{1}=\left(k_{1}-p\right)^{2}, \quad D_{2}=\left(k_{2}-k_{1}\right)^{2}, \ldots, D_{L}=\left(k_{L}-k_{L-1}\right)^{2}, \quad D_{L+1}=\left(k_{L}\right)^{2} .
$$

The polynomial $g(z)$ of this graph can be written in general as

$$
g\left(z_{1}, \ldots, z_{L+1}\right)=\sum_{i=1}^{L+1} \prod_{j \neq i}^{L} z_{j}+s \prod_{i=1}^{L+1} z_{i}
$$

where $s=-p^{2}$. The integral to be computed reads

$$
I(\alpha)=\int_{\mathbb{R}_{+}^{L+1}} \mathrm{~d} \eta_{L+1} \frac{z_{1}^{\alpha_{1}} \cdots z_{L+1}^{\alpha_{L+1}}}{g(z)^{\beta}}
$$

It is easy to check that the $g(z)$ polynomials of this graph lead to codimension zero matrices and hence they fall under the class of problems where we must introduce a deformation to define a GKZ system in the sense of eq. (2.29). In order to perform such deformation systematically, let us introduce some notation. Let $1_{i}$ denote a sequence of 1 's of length $i$ and similarly for $0_{j}$. We have the relation $i+j=L+1$. Furthermore, let

$$
v:=\left(1_{L-1}, 0_{2}\right) .
$$

At each loop, we set a deformation monomial

$$
r(z)=c_{1} z^{v},
$$

hence we have

$$
g_{r}(c, z)=c_{1} z^{v}+\sum_{i=1}^{L+1} c_{L+3-i} \prod_{j \neq i}^{L} z_{j}+c_{L+3} \prod_{i=1}^{L+1} z_{i}
$$


where $c_{L+3}=s$. Let us give an example. For $L=3, v=(1,1,0,0)$ and $r(z)=c_{1} z_{1} z_{2}$, then we have the deformed toric polynomial

$$
g_{r}(c, z)=c_{1} z_{1} z_{2}+c_{2} z_{1} z_{2} z_{3}+c_{3} z_{1} z_{2} z_{4}+c_{4} z_{1} z_{3} z_{4}+c_{5} z_{2} z_{3} z_{4}+c_{6} z_{1} z_{2} z_{3} z_{4} .
$$

After introducing the deformation, the $(L+2) \times(L+3)$ matrix associated with the $L$-loop cantaloupe graph can be written in the general form

$$
A=\left(\begin{array}{ccccc}
1 & 1 & \ldots & 1 & 1 \\
& 1_{L+1} & & 0 & 1_{1} \\
& 1_{L} & & 0 & 1_{2} \\
& & & & \\
& & \vdots & & \\
& & & & \\
& 1_{3} & 0 & & 1_{L-1} \\
0 & 1 & 0 & & 1_{L} \\
0 & 0 & 1 & & 1_{L}
\end{array}\right) .
$$

Notice that each row contains $L+3$ elements. There are $L$ rows with have a single zero and 2 rows with two zeros. The integral under consideration is given by

$$
I_{g_{r}}(\kappa)=\int_{\Omega} \mathrm{d} \eta_{L+1} \frac{z_{1}^{\alpha_{1}} \cdots z_{L+1}^{\alpha_{L+1}}}{g_{r}(c, z)^{\beta}}
$$

where $\kappa=\left(-\beta,-\alpha_{1}, \ldots,-\alpha_{L+1}\right)$. Computing the kernel of the above matrix leads to

$$
\mathcal{L}=\mathbb{Z}\left(1,-1,-1,0_{L-1}, 1\right),
$$

where by definition $0_{0}:=\emptyset$. We choose $w=\left(1,0_{L+2}\right)$, thus obtaining

$$
\operatorname{fin}_{w}\left(H_{\mathrm{A}}(\kappa)\right)=\left\langle\theta_{1} \theta_{L+3}\right\rangle+\left\langle\mathrm{A} \theta-\kappa^{T}\right\rangle .
$$

The roots can be written as

$$
\begin{aligned}
\left\{\gamma_{i}\right\}=\{ & \left(0, \alpha_{L+1}-\beta, \ldots, \alpha_{1}-\beta, L \beta-\sum_{i=1}^{L+1} \alpha_{i}\right), \\
& \left.\left(\sum_{i=1}^{L} \alpha_{i}-L \beta,(L-1) \beta-\sum_{i=1}^{L} \alpha_{i},(L-1) \beta-\sum_{i \neq L}^{L+1} \alpha_{i},-\beta+\alpha_{L-1}, \ldots,-\beta+\alpha_{1}, 0\right)\right\},
\end{aligned}
$$

which lead to the canonical series

$$
\begin{aligned}
& \phi_{1}=c^{\gamma_{1}}{ }_{2} F_{1}\left(\beta-\alpha_{L+1}, \beta-\alpha_{L}, L \beta-\sum_{i=1}^{L+1} \alpha_{i}+1 ; x\right), \\
& \phi_{2}=c^{\gamma_{2}}{ }_{2} F_{1}\left(-(L-1) \beta+\sum_{i=1}^{L} \alpha_{i},-(L-1) \beta+\sum_{i \neq L}^{L+1} \alpha_{i} ; \sum_{i=1}^{L} \alpha_{i}-L \beta+1 ; x\right),
\end{aligned}
$$




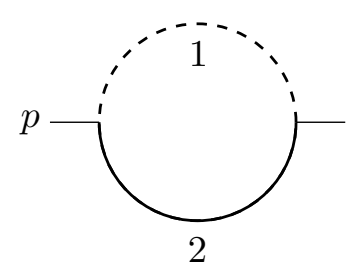

Figure 7. Single mass bubble graph.

where

$$
x=\frac{c_{1} c_{L+3}}{c_{2} c_{3}}
$$

The relevant integration constant reads

$$
K_{1}=\frac{\Gamma\left(-L \beta+\sum_{i=1}^{L+1} \alpha_{i}\right)}{\Gamma(\beta)} \prod_{i=1}^{L+1} \Gamma\left(\beta-\alpha_{i}\right),
$$

which after a change of variables corresponds to the formula of the Mellin transform of a linear function. Setting $c_{1}=0, c_{2}=\cdots=c_{L+2}=1$, and $c_{L+3}=s$ we arrive at

$$
I(\alpha)=s^{\left(L \beta-\sum_{i=1}^{L} \alpha_{i}\right)} \frac{\Gamma\left(-L \beta+\sum_{i=1}^{L+1} \alpha_{i}\right)}{\Gamma(\beta)} \prod_{i=1}^{L+1} \Gamma\left(\beta-\alpha_{i}\right)
$$

The formulas for the fake indicial ideal and its roots have been checked up to 5-loop. In this example, we have seen that deforming $g(z)$ leads to a GKZ system of $\operatorname{co}(\mathrm{A})=1$ and taking the limit of the deformation to zero at the end of the computation allows us to interpret Feynman integrals as the limit of a linear combination of their canonical series.

\subsection{Examples of $\operatorname{co}(\mathrm{A})=1$}

\subsubsection{One-mass bubble}

Let us work now with the bubble integral with one massive internal line (figure 7 ). The inverse propagators of this integral read

$$
D_{1}=\left(k^{2}\right), \quad D_{2}=(k-p)^{2}+m^{2} .
$$

Omitting the overall $\Gamma$ factors we have the Lee-Pomeransky representation

$$
I(\alpha)=\int_{\mathbb{R}_{+}^{2}} \frac{z_{1}^{\alpha_{1}} z_{2}^{\alpha_{2}}}{\left(z_{1}+z_{2}+\left(m^{2}+s\right) z_{1} z_{2}+m^{2} z_{2}^{2}\right)^{\beta}} \frac{\mathrm{d} z_{1}}{z_{1}} \frac{\mathrm{d} z_{2}}{z_{2}},
$$

where $s=-p^{2}$. Therefore, the corresponding toric polynomial and the associated matrix A read

$$
g(c, z)=c_{1} z_{1}+c_{2} z_{2}+c_{3} z_{1} z_{2}+c_{4} z_{2}^{2} \Longleftrightarrow \mathrm{A}=\left(\begin{array}{llll}
1 & 1 & 1 & 1 \\
1 & 0 & 1 & 0 \\
0 & 1 & 1 & 2
\end{array}\right) .
$$


Now, let us consider the related problem from the GKZ point of view. We are interested in the more general situation

$$
I_{g}(\kappa)=\int_{\Omega} \frac{z_{1}^{\alpha_{1}} z_{2}^{\alpha_{2}}}{\left(c_{1} z_{1}+c_{2} z_{2}+c_{3} z_{1} z_{2}+c_{4} z_{2}^{2}\right)^{\beta}} \frac{\mathrm{d} z_{1}}{z_{1}} \frac{\mathrm{d} z_{2}}{z_{2}} .
$$

Taking $w=(0,1,1,1)$, we obtain the following fake indicial ideal

$$
\operatorname{fin}_{w}\left(H_{\mathrm{A}}(\kappa)\right)=\left\langle\theta_{2} \theta_{3}, \beta+\theta_{1}+\theta_{2}+\theta_{3}+\theta_{4}, \alpha_{1}+\theta_{1}+\theta_{3}, \alpha_{2}+\theta_{2}+\theta_{3}+2 \theta_{4}\right\rangle .
$$

Then, the roots of this ideal read

$$
\left\{\gamma_{i}\right\}=\left\{\left(-\alpha_{1}, 2 \alpha_{1}+\alpha_{2}-2 \beta, 0,-\alpha_{1}-\alpha_{2}+\beta\right),\left(\alpha_{1}+\alpha_{2}-2 \beta, 0,-2 \alpha_{1}-\alpha_{2}+2 \beta, \alpha_{1}-\beta\right)\right\} .
$$

In addition, we have $\mathcal{L}=\mathbb{Z}(-1,1,1,-1)$, and hence $u=n(-1,1,1,-1)$. We also have $u . w=n$, which implies $\left[\gamma_{i}\right]_{u_{-}}=0$ for $n<0$. The canonical series simplify to the functions

$$
\begin{aligned}
& \phi_{1}=c^{\gamma_{1}}{ }_{2} F_{1}\left(\alpha_{1}, \alpha_{1}+\alpha_{2}-\beta ; 2 \alpha_{1}+\alpha_{2}-2 \beta+1 ; \frac{c_{2} c_{3}}{c_{1} c_{4}}\right), \\
& \phi_{2}=c^{\gamma_{2}}{ }_{2} F_{1}\left(2 \beta-\alpha_{1}-\alpha_{2}, \beta-\alpha_{1} ; 2 \beta-2 \alpha_{1}-\alpha_{2}+1 ; \frac{c_{2} c_{3}}{c_{1} c_{4}}\right) .
\end{aligned}
$$

The result of the integral is a linear combination of $\phi_{1,2}$. Constants of integration can be obtained by integrating (3.58) taking $c_{3}=0$ and $\Omega=\mathbb{R}_{+}^{2}$, and similarly for $c_{2}$. This leads to the multiplying factors

$$
\begin{aligned}
& K_{1}=\frac{\Gamma\left(\alpha_{1}\right) \Gamma\left(\alpha_{2}+\alpha_{1}-\beta\right) \Gamma\left(2 \beta-2 \alpha_{1}-\alpha_{2}\right)}{\Gamma(\beta)}, \\
& K_{2}=\frac{\Gamma\left(\beta-\alpha_{1}\right) \Gamma\left(2 \alpha_{1}+\alpha_{2}-2 \beta\right) \Gamma\left(2 \beta-\alpha_{1}-\alpha_{2}\right)}{\Gamma(\beta)} .
\end{aligned}
$$

Setting $c_{3}=\left(s+m^{2}\right)$ and $c_{4}=m^{2}$, the resulting integral reads

$$
\begin{aligned}
I(\alpha)= & \left(m^{2}\right)^{\beta-\alpha_{1}-\alpha_{2}}\left(K_{1}{ }_{2} F_{1}\left(\alpha_{1}, \alpha_{1}+\alpha_{2}-\beta ; 2 \alpha_{1}+\alpha_{2}-2 \beta+1 ; 1+s / m^{2}\right)\right. \\
& \left.+\left(1+s / m^{2}\right)^{2 \beta-2 \alpha_{1}-\alpha_{2}} K_{2}{ }_{2} F_{1}\left(2 \beta-\alpha_{1}-\alpha_{2}, \beta-\alpha_{1} ; 2 \beta-2 \alpha_{1}-\alpha_{2}+1 ; 1+s / m^{2}\right)\right) .
\end{aligned}
$$

The form of the result as a sum of two hypergeometric functions is reminiscent of the negative dimension approach [62]. Using the eq. (A.8), we obtain

$$
\begin{aligned}
I(\alpha)= & \left(m^{2}\right)^{\beta-\alpha_{1}-\alpha_{2}} \frac{\Gamma\left(\alpha_{1}\right) \Gamma\left(\beta-\alpha_{1}\right) \Gamma\left(2 \beta-\alpha_{1}-\alpha_{2}\right) \Gamma\left(-\beta+\alpha_{1}+\alpha_{2}\right)}{\Gamma(\beta)^{2}} \\
& \times{ }_{2} F_{1}\left(\alpha_{1},-\beta+\alpha_{1}+\alpha_{2} ; \beta ;-s / m^{2}\right) .
\end{aligned}
$$

\subsubsection{One-mass sunset}

We now consider the single mass sunset graph with the constraint $s=-p^{2}=m^{2}$ (figure 8). The inverse propagators read

$$
D_{1}=\left(k_{1}-p_{1}\right)^{2}, \quad D_{2}=\left(k_{2}-k_{1}\right)^{2}+m^{2}, \quad D_{3}=k_{2}^{2},
$$




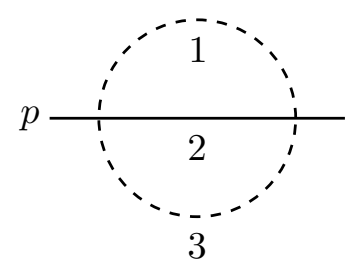

Figure 8. Sunset graph.

which lead to the integral

$$
I(\alpha)=\int_{\mathbb{R}_{+}^{3}} \mathrm{~d} \eta_{3} \frac{z_{1}^{\alpha_{1}} z_{2}^{\alpha_{2}} z_{3}^{\alpha_{3}}}{\left(z_{1} z_{2}+z_{1} z_{3}+z_{2} z_{3}+m^{2} z_{1} z_{2}^{2}+m^{2} z_{2}^{2} z_{3}\right)^{\beta}} .
$$

We consider the following toric polynomial

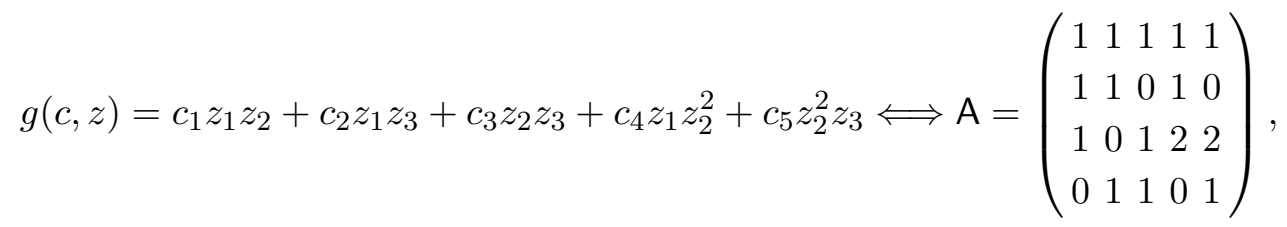

such that the integral under consideration becomes

$$
I_{g}(\kappa)=\int_{\Omega} \mathrm{d} \eta_{3} \frac{z_{1}^{\alpha_{1}} z_{2}^{\alpha_{2}} z_{3}^{\alpha_{3}}}{\left(c_{1} z_{1} z_{2}+c_{2} z_{1} z_{3}+c_{3} z_{2} z_{3}+c_{4} z_{1} z_{2}^{2}+c_{5} z_{2}^{2} z_{3}\right)^{\beta}} .
$$

Choosing the weight vector $w=(0,1,1,1,1)$, we find

$$
\begin{aligned}
\operatorname{fin}_{w}\left(H_{\mathrm{A}}(\kappa)\right)= & \left\langle\theta_{3} \theta_{4}\right\rangle+\left\langle\mathrm{A} \theta-\kappa^{T}\right\rangle, \\
\left\{\gamma_{i}\right\}= & \left\{\left(\alpha_{3}-\beta,-\alpha_{1}-\alpha_{3}+\beta, 2 \alpha_{1}+\alpha_{2}+\alpha_{3}-3 \beta, 0,-\alpha_{1}-\alpha_{2}-\alpha_{3}+2 \beta\right),\right. \\
& \left.\left(2 \alpha_{1}+\alpha_{2}+2 \alpha_{3}-4 \beta,-\alpha_{1}-\alpha_{3}+\beta, 0,-2 \alpha_{1}-\alpha_{2}-\alpha_{3}+3 \beta, \alpha_{1}-\beta\right)\right\} .
\end{aligned}
$$

From $\mathcal{L}=\mathbb{Z}(1,0,-1,-1,1)$, we have $u=n(1,0,-1,-1,1)$, hence $u . w=-n$. In order to start the sum from $n=0$, we set $u \rightarrow-u$ and thus $u=n(0,0,1,1,0)-n(1,0,0,0,1)$. We obtain

$$
\begin{aligned}
& \phi_{1}=c^{\gamma_{1}} \sum_{n \geq 0} \frac{\left(\beta-\alpha_{3}\right)_{n}\left(\alpha_{1}+\alpha_{2}+\alpha_{3}-2 \beta\right)_{n}}{\left(2 \alpha_{1}+\alpha_{2}+\alpha_{3}-3 \beta+1\right)_{n}(1)_{n}}\left(\frac{c_{3} c_{4}}{c_{1} c_{5}}\right)^{n}, \\
& \phi_{2}=c^{\gamma_{2}} \sum_{n \geq 0} \frac{\left(-2 \alpha_{1}-\alpha_{2}-2 \alpha_{3}+4 \beta\right)_{n}\left(\beta-\alpha_{1}\right)_{n}}{(1)_{n}\left(-2 \alpha_{1}-\alpha_{2}-\alpha_{3}+3 \beta+1\right)_{n}}\left(\frac{c_{3} c_{4}}{c_{1} c_{5}}\right)^{n} .
\end{aligned}
$$

The integration constants can be written collectively as

$$
K_{r}=\frac{1}{\Gamma(\beta)} \prod_{i \neq 0} \Gamma\left(-\gamma_{r}^{i}\right) .
$$




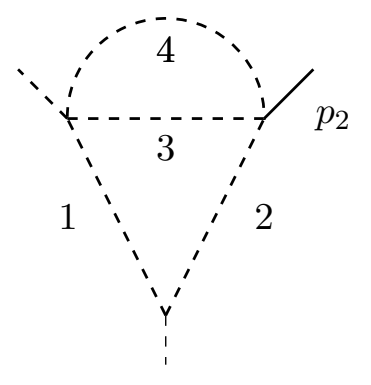

Figure 9. Party hat.

Finally, setting $c_{1}=c_{2}=c_{3}=1$ and $c_{4}=c_{5}=m^{2}$, we arrive at the result

$$
\begin{aligned}
I(\alpha)= & \left(m^{2}\right)^{-\alpha_{1}-\alpha_{2}-\alpha_{3}+2 \beta}\left(K_{12} F_{1}\left(\beta-\alpha_{3}, \alpha-2 \beta ; \alpha+\alpha_{1}-3 \beta+1 ; 1\right)\right. \\
& \left.+K_{2}{ }_{2} F_{1}\left(-2 \alpha+\alpha_{2}+4 \beta, \beta-\alpha_{1} ;-\alpha-\alpha_{1}+3 \beta+1 ; 1\right)\right) .
\end{aligned}
$$

In order to write this result in simpler form let

$$
A=\beta-\alpha_{3}, \quad B=-2 \beta+\alpha_{1}+\alpha_{2}+\alpha_{3}, \quad C=2 \beta-\alpha_{1}-\alpha_{3},
$$

hence we can write eq. (3.76) as

$$
I(\alpha)=\left(m^{2}\right)^{-B} \frac{\Gamma(A) \Gamma(B) \Gamma(\beta-C) \Gamma(C-A) \Gamma(C-B)}{\Gamma(C) \Gamma(\beta)},
$$

where we have used identity (A.8).

\subsubsection{Single scale party hat}

The next example is the party hat graph shown in figure 9 . We have the inverse propagators

$$
D_{1}=k_{2}^{2}, \quad D_{2}=\left(k_{2}-p_{3}\right)^{2}, \quad D_{3}=\left(k_{2}-k_{1}\right)^{2}, \quad D_{4}=\left(k_{1}-p_{1}\right)^{2} .
$$

We will consider the case $-p_{1}^{2}=-p_{3}^{2}=0$ and $s=p_{2}^{2}$. The relevant integral in the Lee-Pomeransky representation reads

$$
I(\alpha)=\int_{\mathbb{R}_{+}^{4}} \mathrm{~d} \eta_{4} \frac{z_{1}^{\alpha_{1}} z_{2}^{\alpha_{2}} z_{3}^{\alpha_{3}} z_{4}^{\alpha_{4}}}{\left(z_{1} z_{3}+z_{1} z_{4}+z_{2} z_{3}+z_{2} z_{4}+z_{4} z_{3}+s z_{2} z_{4} z_{3}\right)^{\beta}} .
$$

Therefore, the toric polynomial has the form

$$
g(c, z)=c_{1} z_{1} z_{3}+c_{2} z_{1} z_{4}+c_{3} z_{2} z_{3}+c_{4} z_{2} z_{4}+c_{5} z_{3} z_{4}+c_{6} z_{2} z_{3} z_{4},
$$

where $c_{6}=s$. We associate the following matrix to $g(c, z)$

$$
A=\left(\begin{array}{llllll}
1 & 1 & 1 & 1 & 1 & 1 \\
1 & 1 & 0 & 0 & 0 & 0 \\
0 & 0 & 1 & 1 & 0 & 1 \\
1 & 0 & 1 & 0 & 1 & 1 \\
0 & 1 & 0 & 1 & 1 & 1
\end{array}\right)
$$


Taking $w=(0,1,1,1,1,1)$ leads to the fake indicial ideal (see example appendix B)

$$
\begin{aligned}
\operatorname{fin}_{w}\left(H_{\mathrm{A}}(\kappa)\right)= & \left\langle\theta_{2} \theta_{3}, \beta+\theta_{1}+\theta_{2}+\theta_{3}+\theta_{4}+\theta_{5}+\theta_{6}, \alpha_{1}+\theta_{1}+\theta_{2}, \alpha_{2}+\theta_{3}+\theta_{4}+\theta_{6}\right. \\
& \left.\alpha_{3}+\theta_{1}+\theta_{3}+\theta_{5}+\theta_{6}, \alpha_{4}+\theta_{2}+\theta_{4}+\theta_{5}+\theta_{6}\right\rangle
\end{aligned}
$$

with roots

$$
\begin{aligned}
\gamma_{i}= & \left\{\left(-\alpha_{1}, 0, \alpha_{1}+\alpha_{4}-\beta, \alpha_{3}-\beta, \alpha_{1}+\alpha_{2}-\beta,-\alpha_{1}-\alpha_{2}-\alpha_{3}-\alpha_{4}+2 \beta\right),\right. \\
& \left.\left(\alpha_{4}-\beta,-\alpha_{1}-\alpha_{4}+\beta, 0, \alpha_{1}+\alpha_{3}+\alpha_{4}-2 \beta, \alpha_{1}+\alpha_{2}-\beta,-\alpha_{1}-\alpha_{2}-\alpha_{3}-\alpha_{4}+2 \beta\right)\right\} .
\end{aligned}
$$

Computing $\operatorname{ker}(\mathrm{A})$ leads to

$$
\mathcal{L}=\mathbb{Z}(1,-1,-1,1,0,0) \Rightarrow u=n(1,0,0,1,0,0)-n(0,1,1,0,0,0) .
$$

Since $u . w=-n,[\gamma]_{u_{-}}=0$ for $n>0$. Then, the canonical series (2.29) leads to

$$
\begin{aligned}
& \phi_{1}=c^{\gamma_{1}}{ }_{2} F_{1}\left(\alpha_{1}, \beta-\alpha_{3} ; \alpha_{1}+\alpha_{4}-\beta+1 ;\left(c_{1} c_{4}\right) /\left(c_{2} c_{5}\right)\right), \\
& \phi_{2}=c^{\gamma_{2}}{ }_{2} F_{1}\left(\beta-\alpha_{4},-\alpha_{1}-\alpha_{3}-\alpha_{4}+2 \beta ;-\alpha_{1}-\alpha_{4}+\beta+1 ;\left(c_{2} c_{4}\right) /\left(c_{1} c_{5}\right)\right) .
\end{aligned}
$$

Integration constants read

$$
K_{r}=\frac{1}{\Gamma(\beta)} \prod_{i \neq 0} \Gamma\left(-\gamma_{r}^{i}\right) .
$$

Setting $c_{1}=c_{2}=c_{3}=c_{4}=c_{5}=1, c_{6}=s$, and defining

$$
A=\beta-\alpha_{4}, \quad B=2 \beta-\alpha_{1}-\alpha_{3}-\alpha_{4}, \quad C=2 \beta-\alpha_{3}-\alpha_{4},
$$

we arrive at

$$
I(\alpha)=s^{B-\alpha_{2}} \frac{\Gamma(A) \Gamma(B) \Gamma(C-A) \Gamma\left(\alpha_{2}-B\right) \Gamma(C-B) \Gamma\left(B-C+\beta-\alpha_{2}\right)}{\Gamma(C) \Gamma(\beta)},
$$

where we have used identity (A.8).

\subsubsection{On-shell massless box}

Let us now consider the massless box integral shown in figure 10. The inverse propagators are given by

$$
D_{1}=\left(k-p_{1}\right)^{2}, \quad D_{2}=\left(k+p_{2}+p_{3}\right)^{2}, \quad D_{3}=\left(k+p_{2}\right)^{2} \quad D_{4}=k_{1}^{2},
$$

where $-p_{i}^{2}=0, i=1, \ldots, 4$. We obtain

$$
g(z)=z_{1}+z_{2}+z_{3}+z_{4}+s z_{1} z_{3}+t z_{2} z_{4},
$$

where $s=-\left(p_{1}+p_{2}\right)^{2}$ and $t=-\left(p_{2}+p_{3}\right)^{2}$ are the usual Mandelstam invariants. From this polynomial we obtain the matrix

$$
\mathrm{A}=\left(\begin{array}{llllll}
1 & 1 & 1 & 1 & 1 & 1 \\
1 & 0 & 0 & 0 & 1 & 0 \\
0 & 1 & 0 & 0 & 0 & 1 \\
0 & 0 & 1 & 0 & 1 & 0 \\
0 & 0 & 0 & 1 & 0 & 1
\end{array}\right)
$$




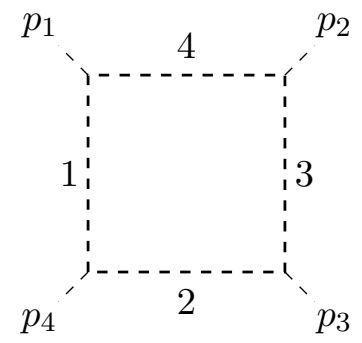

Figure 10. On shell massless box.

Let us consider the more general problem

$$
I_{g}(\kappa)=\int_{\Omega} \frac{z_{1}^{\alpha_{1}} z_{2}^{\alpha_{2}} z_{3}^{\alpha_{3}} z_{4}^{\alpha_{4}}}{\left(c_{1} z_{1}+c_{2} z_{2}+c_{3} z_{3}+c_{4} z_{4}+c_{5} z_{3} z_{1}+c_{6} z_{2} z_{4}\right)^{\beta}} \mathrm{d} \eta_{4} .
$$

Choosing $w=(0,1,0,0,0,0)$, the fake indicial ideal reads

$$
\operatorname{fin}_{w}\left(H_{\mathrm{A}}(\kappa)\right)=\left\langle\theta_{2} \theta_{4} \theta_{5}\right\rangle+\left\langle\mathrm{A} \theta-\kappa^{T}\right\rangle,
$$

which lead to the roots

$$
\begin{aligned}
\left\{\gamma_{i}\right\}= & \left\{\left(-\alpha_{1}, \alpha_{1}+\alpha_{3}+\alpha_{4}-\beta,-\alpha_{3}, \alpha_{1}+\alpha_{2}+\alpha_{3}-\beta, 0,-\alpha_{1}-\alpha_{2}-\alpha_{3}-\alpha_{4}+\beta\right),\right. \\
& \left(\alpha_{2}+\alpha_{3}-\beta, \alpha_{4}-\alpha_{2}, \alpha_{1}+\alpha_{2}-\beta, 0,-\alpha_{1}-\alpha_{2}-\alpha_{3}+\beta,-\alpha_{4}\right), \\
& \left.\left(\alpha_{3}+\alpha_{4}-\beta, 0, \alpha_{1}+\alpha_{4}-\beta, \alpha_{2}-\alpha_{4},-\alpha_{1}-\alpha_{3}-\alpha_{4}+\beta,-\alpha_{2}\right)\right\}
\end{aligned}
$$

Computing ker A leads to $u=n(-1,1,-1,1,1,-1)$, hence $u . w=n$. Therefore $[\gamma]_{u_{-}}=0$ for $n<0$. We also have

$$
u_{-}=(n, 0, n, 0,0, n), \quad u_{+}=(0, n, 0, n, n, 0) .
$$

We can now write the canonical series (2.29) as

$$
\begin{aligned}
& \phi_{1}=c^{\gamma_{1}} \sum_{n \geq 0} \frac{\left(\alpha_{1}\right)_{n}\left(\alpha_{3}\right)_{n}\left(-\beta+\alpha_{1}+\alpha_{2}+\alpha_{3}+\alpha_{4}\right)_{n}}{(1)_{n}\left(-\beta+\alpha_{1}+\alpha_{2}+\alpha_{3}+1\right)_{n}\left(-\beta+\alpha_{1}+\alpha_{3}+\alpha_{4}+1\right)_{n}}\left(\frac{c_{2} c_{4} c_{5}}{c_{1} c_{3} c_{6}}\right)^{n}, \\
& \phi_{2}=c^{\gamma_{2}} \sum_{n \geq 0} \frac{\left(\alpha_{4}\right)_{n}\left(\beta-\alpha_{1}-\alpha_{2}\right)_{n}\left(\beta-\alpha_{2}-\alpha_{3}\right)_{n}}{(1)_{n}\left(-\alpha_{2}+\alpha_{4}+1\right)_{n}\left(\beta-\alpha_{1}-\alpha_{2}-\alpha_{3}+1\right)_{n}}\left(\frac{c_{2} c_{4} c_{5}}{c_{1} c_{3} c_{6}}\right)^{n} \\
& \phi_{3}=c^{\gamma_{3}} \sum_{n \geq 0} \frac{\left(\alpha_{2}\right)_{n}\left(\beta-\alpha_{1}-\alpha_{4}\right)_{n}\left(\beta-\alpha_{3}-\alpha_{4}\right)_{n}}{(1)_{n}\left(\alpha_{2}-\alpha_{4}+1\right)_{n}\left(\beta-\alpha_{1}-\alpha_{3}-\alpha_{4}+1\right)_{n}}\left(\frac{c_{2} c_{4} c_{5}}{c_{1} c_{3} c_{6}}\right)^{n} .
\end{aligned}
$$

The constants of integration read

$$
K_{r}=\frac{1}{\Gamma(\beta)} \prod_{i \neq 0} \Gamma\left(-\gamma_{r}^{i}\right)
$$

In this case the canonical series evaluate to hypergeometric functions ${ }_{3} F_{2}(a, b ; c ; d, e ; x)$ (see definition in eq. (A.4)). Let $A=\alpha_{1}+\alpha_{2}+\alpha_{3}+\alpha_{4}$ and $x=\left(c_{2} c_{4} c_{5}\right) /\left(c_{1} c_{3} c_{6}\right)$. We can 


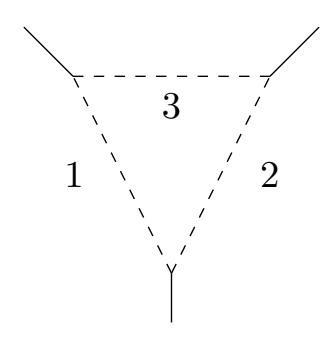

Figure 11. Three-scale triangle: $s_{1}=-p_{1}^{2}, s_{2}=-p_{2}^{2}, s_{3}=-\left(p_{1}+p_{2}\right)^{2}$.

write the result in the condensed form

$$
\begin{aligned}
I_{b}(\kappa, c)= & K_{1} c^{\gamma_{1}}{ }_{3} F_{2}\left(\alpha_{1}, \alpha_{3},-\beta+A ;-\beta+A-\alpha_{4}+1,-\beta+A-\alpha_{2}+1 ; x\right) \\
& +K_{2} c^{\gamma_{2}}{ }_{3} F_{2}\left(\beta-\alpha_{1}-\alpha_{2}, \beta-\alpha_{2}-\alpha_{3}, \alpha_{4} ; \beta-A+\alpha_{4}+1,-\alpha_{2}+\alpha_{4}+1 ; x\right) \\
& +K_{3} c^{\gamma_{3}}{ }_{3} F_{2}\left(\alpha_{2}, \beta-\alpha_{1}-\alpha_{4}, \beta-\alpha_{3}-\alpha_{4} ; \alpha_{2}-\alpha_{4}+1, \beta-A+\alpha_{2}+1 ; x\right)
\end{aligned}
$$

Setting the constant to the values of the original polynomial, i.e., $c_{1}=\cdots=c_{4}=1, c_{5}=s$ and $c_{6}=t$, we obtain

$$
\begin{aligned}
I(\alpha)= & K_{1} t^{\beta-A_{3}} F_{2}\left(\alpha_{1}, \alpha_{3},-\beta+A ;-\beta+A-\alpha_{4}+1,-\beta+A-\alpha_{2}+1 ; s / t\right) \\
& +K_{2} t^{-\alpha_{4}} s^{\alpha_{4}-A+\beta}{ }_{3} F_{2}\left(\beta-\alpha_{1}-\alpha_{2}, \beta-\alpha_{2}-\alpha_{3}, \alpha_{4} ; \beta-A+\alpha_{4}+1,-\alpha_{2}+\alpha_{4}+1 ; s / t\right) \\
& +K_{3} t^{-\alpha_{2}} s^{\alpha_{2}-A+\beta}{ }_{3} F_{2}\left(\alpha_{2}, \beta-\alpha_{1}-\alpha_{4}, \beta-\alpha_{3}-\alpha_{4} ; \alpha_{2}-\alpha_{4}+1, \beta-A+\alpha_{2}+1 ; s / t\right) .
\end{aligned}
$$

Hence, we have recovered the result obtained by the differential reduction method using Gröbner bases given in ref. [63] based on [64, 65]. Interestingly, the computation of integrations constants in ref. [63] follows a similar prescription as ours. However, in our case, because of our choice of $w$, we only set $c_{5}=0$ to compute $K_{1}$ but we do not use $c_{6}=0$ as in [63]. This results also matches nicely the results obtained in ref. [66] using the negative dimension approach.

\subsection{A $\operatorname{co}(\mathrm{A})=2$ example}

Finally, let us consider the triangle integral shown in figure 11 where all external momenta are nonvanishing. The inverse propagators read

$$
D_{1}=\left(k_{1}-p_{1}\right)^{2}, \quad D_{2}=\left(k_{1}+p_{2}\right), \quad D_{3}=k_{1}^{2},
$$

where we have $-p_{1}^{2}=s_{1},-p_{2}^{2}=s_{2}$, and $-\left(p_{1}+p_{2}\right)^{2}=s_{3}$. Using momentum conservation, we have

$$
g(z)=z_{1}+z_{2}+z_{3}+s_{3} z_{1} z_{2}+s_{1} z_{1} z_{3}+s_{2} z_{2} z_{3} .
$$

The associated matrix reads

$$
A=\left(\begin{array}{llllll}
1 & 1 & 1 & 1 & 1 & 1 \\
1 & 0 & 0 & 1 & 1 & 0 \\
0 & 1 & 0 & 1 & 0 & 1 \\
0 & 0 & 1 & 0 & 1 & 1
\end{array}\right)
$$


This matrix has $\operatorname{co}(\mathrm{A})=2$ and hence the resulting A-hypergeometric functions will be functions of two variables. The integral under consideration then reads

$$
I_{g}(\kappa)=\int_{\Omega} \mathrm{d} \eta_{3} \frac{z_{1}^{\alpha_{1}} z_{2}^{\alpha_{2}} z_{3}^{\alpha_{3}}}{\left(c_{1} z_{1}+c_{2} z_{2}+c_{3} z_{3}+c_{4} z_{1} z_{2}+c_{5} z_{1} z_{3}+c_{6} z_{2} z_{3}\right)^{\beta}} .
$$

Computing $\operatorname{ker}(\mathrm{A})$, we have

$$
\mathcal{L}=\{(-1,0,1,1,0,-1),(-1,1,0,0,1,-1)\},
$$

which means that in this case the lattice is generated by

$$
u=m(-1,0,1,1,0,-1)+n(-1,1,0,0,1,-1), \quad m, n \in \mathbb{Z} .
$$

We choose $w=(0,0,1,0,0,0)$, which has the advantage of restricting the sum over $m$. We have $u . w=m$, hence $\left[\gamma_{i}\right]_{u_{-}}=0$ for $m<0$. The fake indicial ideal reads

$$
\operatorname{fin}_{w}\left(H_{\mathrm{A}}(\kappa)\right)=\left\langle\theta_{2} \theta_{5}, \theta_{3} \theta_{4}\right\rangle+\left\langle\mathrm{A} \theta-\kappa^{T}\right\rangle
$$

with roots

$$
\begin{aligned}
&\left\{\gamma_{i}\right\}=\left\{\left(-\alpha_{1}, C-\beta, B-\beta, 0,0, \beta-A\right),\left(\alpha_{2}-\beta, C-\beta, 0, \beta-B, 0,-\alpha_{3}\right),\right. \\
&\left.\left(\alpha_{3}-\beta, 0, B-\beta, 0, \beta-C,-\alpha_{2}\right),\left(A-2 \beta, 0,0, \beta-B, \beta-C, \alpha_{1}-\beta\right)\right\}
\end{aligned}
$$

where $A=\alpha_{1}+\alpha_{2}+\alpha_{3}, B=\alpha_{1}+\alpha_{2}$, and $C=\alpha_{1}+\alpha_{3}$. Inserting the roots in eq. (2.29) gives four solutions

$$
\begin{aligned}
& \phi_{1}=c^{\gamma_{1}} \sum_{m \geq 0, n \in \mathbb{Z}} \frac{\left(\alpha_{1}\right)_{m+n}(A-\beta)_{m+n}}{(-\beta+C+1)_{n}(-\beta+B+1)_{m}(1)_{m}(1)_{n}} x^{m} y^{n}, \\
& \phi_{2}=c^{\gamma_{2}} \sum_{m \geq 0, n \in \mathbb{Z}} \frac{\left(\beta-\alpha_{2}\right)_{m+n}\left(\alpha_{3}\right)_{m+n}}{(-\beta+C+1)_{n}(1)_{m}(\beta-B+1)_{m}(1)_{n}} x^{m} y^{n}, \\
& \phi_{3}=c^{\gamma_{3}} \sum_{m \geq 0, n \in \mathbb{Z}} \frac{\left(\beta-\alpha_{3}\right)_{m+n}\left(\alpha_{2}\right)_{m+n}}{(1)_{n}(-\beta+B+1)_{m}(1)_{m}(\beta-C+1)_{n}} x^{m} y^{n}, \\
& \phi_{4}=c^{\gamma_{4}} \sum_{m \geq 0, n \in \mathbb{Z}} \frac{(2 \beta-A)_{m+n}\left(\beta-\alpha_{1}\right)_{m+n}}{(1)_{n}(1)_{m}(\beta-B+1)_{m}(\beta-C+1)_{n}} x^{m} y^{n},
\end{aligned}
$$

where we have defined $x=\left(c_{3} c_{4}\right) /\left(c_{1} c_{6}\right)$ and $y=\left(c_{2} c_{5}\right) /\left(c_{1} c_{6}\right)$. Due to our choice of weight vector, the sum has been only restricted in $m$. However, it is easy to see that the sum over negative integers $n$ vanish due to the presence of $(1)_{n}$. Hence the above sums have the form

$$
\sum_{m, n \geq 0} \frac{(a)_{m+n}(b)_{m+n}}{(c)_{m}(d)_{n}(1)_{m}\left(1_{n}\right)} x^{m} y^{n}
$$

which are sum representations of the Appell hypergeometric function $F_{4}$ (see eq. (A.5)). In order to compute the integration constants we can follow our prescription and set $c_{4}=c_{5}=0$ in eq. (3.106) and take $\Omega=\mathbb{R}^{3}$. This computes the first coefficient in the expansion $K_{1}$. We 
follow the same procedure for the remaining integration constants. They can can collectively be written as

$$
K_{r}=\frac{1}{\Gamma(\beta)} \sum_{i \neq 0} \Gamma\left(-\gamma_{r}^{i}\right)
$$

Setting $c_{1}=c_{2}=c_{3}=1$ and $c_{4}=s_{3}, c_{5}=s_{1}$, and $c_{6}=s_{2}$, we can write the Feynman integral as

$$
\begin{aligned}
I(\alpha)= & K_{1} s_{2}^{\beta-A} F_{4}\left(\alpha_{1}, A-\beta ;-\beta+\alpha_{13}+1,-\beta+\alpha_{12}+1 ; s_{3} / s_{2}, s_{1} / s_{2}\right) \\
& +K_{2} s_{2}^{-\alpha_{3}} s_{3}^{\beta-B} F_{4}\left(\beta-\alpha_{2}, \alpha_{3} ; C-\beta+1,-B+\beta+1 ; s_{3} / s_{2}, s_{1} / s_{2}\right) \\
& +K_{3} s_{2}^{-\alpha_{2}} s_{1}^{\beta-C} F_{4}\left(\beta-\alpha_{3}, \alpha_{2} ; B-\beta+1,-C+\beta+1 ; s_{3} / s_{2}, s_{1} / s_{2}\right) \\
& +K_{4} s_{1}^{\beta-C} s_{2}^{\alpha_{1}-\beta} s_{3}^{\beta-B} F_{4}\left(2 \beta-A, \beta-\alpha_{1} ;-B+\beta+1,-C+\beta+1 ; s_{3} / s_{2}, s_{1} / s_{2}\right),
\end{aligned}
$$

which agrees with the results obtained via the Mellin-Barnes integral representations [67, 68] and the negative dimension approach [62].

\section{Conclusions and outlook}

In this paper have studied the relation between the Lee-Pomeransky representation of Feynman integrals and GKZ systems. We have shown that in generic cases we can associate a matrix $\mathrm{A}$ of $\operatorname{co}(\mathrm{A})>0$ to a deformed polynomial $g_{r}(c, z)=r(c, z)+\mathcal{U}(c)+\mathcal{F}(c)$, where $r(c, z)$ is introduced to ensure a canonical series representation. $\mathcal{U}(c)$ and $\mathcal{F}(c)$ are toric polynomials associated with the Symanzik polynomials. Under these restrictions, we can interpret a large class of Feynman integrals as furnishing a solution of a GKZ system based on A. The canonical series algorithm then allows us to evaluate integrals with arbitrary powers in the propagators as linear combinations of A-hypergeometric functions. Feynman integrals are recovered at the end of the computation by identifying the coefficients of the toric polynomials with their kinematic values.

Using the canonical series method, we have evaluated several integrals for arbitrary noninteger powers in the propagators. A particularly nontrivial example is the on-shell massless box with arbitrary powers in the propagators. With this method the result was obtained as a particular case of an A-hypergeometric integral and it matches the result based on the recurrence relations method based on Gröbner bases [63] and the negative dimension approach [66]. Another nontrivial example is the $\operatorname{co}(\mathrm{A})=2$ example of the three-scale massless triangle, which is in agreement with the negative dimension approach as well. It would be interesting to study the relation between those methods and the canonical series algorithm.

Computing canonical series is a straightforward computational algebra problem. However, these series heavily depend on the choice of a weight vector $w$, which sets the initial ideal and effectively chooses a domain of convergence. This choice is tied with the available information about the integration cycle and ultimately with our ability to compute integration constants. Our recipe of setting $\Omega=\mathbb{R}_{+}^{N}$ is the obvious choice and was motivated by the cycles, which one obtains by studying the coamoeba of $g_{r}(z)$ in Euclidean kinematics. 
A full characterization of the coamoeba of $g_{r}(z)$ might be necessary when non-Euclidean kinematics is considered and going to higher codimensions.

The rank of the system arising from the toric version of $g_{r}(z)$ is bounded by $\operatorname{vol}(\mathrm{A})$ which, in general, is greater than the number of master integrals arising from IBP identities or from the Euler characteristic [69]. The canonical series algorithm produces vol(A) hypergeometric series in $\operatorname{co}(\mathrm{A})$ variables. They collapse to simpler expressions once we set the coefficients $c$ to their kinematic values. This typically involves setting one or more of these variables to unity, which amounts to evaluate hypergeometric functions at singular points. At $\operatorname{co}(A)=1$ the functions appearing at those limits are $\Gamma$-functions. At $\operatorname{co}(A)=2$, those limits lead to hypergeometric functions of one variable, which then collapse to simpler expressions. It would be interesting to study the mechanism which relates the number of master integrals and the number of canonical series solutions.

We believe that the application of GKZ systems and canonical series to Feynman integrals is not limited to the Lee-Pomeransky representation. Indeed, it would be interesting to apply these ideas in representations where an algebraic definition of the integration cycles is available. For instance, this is the case of the representation due to Baikov [70], which has recently been studied specially in the context of maximal cuts [71-75]. This approach is closely related to ref. [76], where bases of Pfaffian systems for GKZ systems are constructed using twisted cohomology groups.

We leave these explorations for future work.

\section{Acknowledgments}

We thank Einan Gardi for useful discussions and encouragement during the realization of this project. We thank Jens Forsgård for providing his MATHEMATICA notebook for drawing coamoebas. We thank Claude Duhr for useful comments. We thank the anonymous referee for useful comments. We thank the Galileo Galilei Institute Institute in Florence for hospitality and partial financial support for the workshop "Amplitudes in the LHC era" in Autumn 2018. We thank the ETH Institute for Theoretical Studies in the framework of the program "Periods, modular forms and scattering amplitudes" for hospitality and partial financial support. The author's research is supported by the STFC Consolidated Grant 'Particle Physics at the Higgs Centre'.

\section{A Useful formulas}

\section{Integrals.}

$$
\begin{aligned}
& \int_{\mathbb{R}_{+}} \frac{z^{\alpha}}{(a+b z)^{\beta}} \frac{d z}{z}=\frac{\Gamma(\beta-\alpha) \Gamma(\alpha)}{\Gamma(\beta)} a^{\alpha-\beta} b^{-\alpha}, \\
& \operatorname{Re}(\alpha)>0, \quad \operatorname{Re}(\beta-\alpha)>0 . \\
& \int_{\mathbb{R}_{+}} \frac{z^{\alpha}}{(1+z)^{\beta_{1}}(1+c z)^{\beta_{2}}} \frac{d z}{z}=\frac{\Gamma\left(\beta_{1}+\beta_{2}-\alpha\right) \Gamma(\alpha)}{\Gamma\left(\beta_{1}+\beta_{2}\right)}{ }_{2} F_{1}\left(\alpha, \beta_{2} ; \beta_{1}+\beta_{2} ; 1-c\right), \\
& \operatorname{Re}\left(\beta_{1}+\beta_{2}\right)>\operatorname{Re}(\alpha)>0, \quad|\arg c|<\pi
\end{aligned}
$$


Sum representations.

$$
\begin{aligned}
{ }_{2} F_{1}(a, b, c ; x) & =\sum_{n \geq 0} \frac{(a)_{n}(b)_{n}}{(c)_{n}(1)_{n}} x^{n}, \\
{ }_{3} F_{2}(a, b, c ; d, e ; x) & =\sum_{n \geq 0} \frac{(a)_{n}(b)_{n}(c)_{n}}{(d)_{n}(e)_{n}(1)_{n}} x^{n}, \\
F_{4}(a, b ; c, d ; x, y) & =\sum_{m, n \geq 0} \frac{(a)_{m+n}(b)_{m+n}}{(c)_{m}(d)_{n}(1)_{m}\left(1_{n}\right)} x^{m} y^{n} .
\end{aligned}
$$

\section{Linear transformations.}

$$
\begin{aligned}
{ }_{2} F_{1}(a, b, c ; 1)= & \frac{\Gamma(c) \Gamma(c-a-b)}{\Gamma(c-a) \Gamma(c-b)}, \\
{ }_{2} F_{1}(a, b, c ; z)= & (1-z)^{(c-a-b)}{ }_{2} F_{1}(c-b, c-a ; c ; z), \\
{ }_{2} F_{1}(a, b, c ; z)= & \frac{\Gamma(c) \Gamma(c-a-b)}{\Gamma(c-a) \Gamma(c-b)}{ }_{2} F_{1}(a, b ; a+b-c+1 ; 1-z) \quad(|\arg (1-z)|<\pi) \quad(\text { A. } 8) \\
& +(1-z)^{c-a-b} \frac{\Gamma(c) \Gamma(a+b-c)}{\Gamma(a) \Gamma(b)}{ }_{2} F_{1}(c-a, c-b ; c-a-b+1 ; 1-z), \\
{ }_{2} F_{1}(a, b, c ; z)= & (1-z)^{-a} \frac{\Gamma(c) \Gamma(b-a)}{\Gamma(b) \Gamma(c-a)}{ }_{2} F_{1}\left(a, c-b ; a-b+1 ; \frac{1}{1-z}\right) \quad(|\arg (1-z)|<\pi) \\
& +(1-z)^{-b} \frac{\Gamma(c) \Gamma(a-b)}{\Gamma(a) \Gamma(c-b)}{ }_{2} F_{1}\left(b, c-a ; b-a+1 ; \frac{1}{1-z}\right) .
\end{aligned}
$$

\section{Pochhammer identities.}
$(0)_{0}=1$,
$(0)_{m}=0$,
$m \in \mathbb{N}$,
$(a)_{-m}=\frac{(-1)^{m}}{(1-a)_{m}}$,
${ }^{(0)}{ }_{-m}=\frac{(-1)^{m}}{(1)_{m}}$,
$m \in \mathbb{N}$,
$(a)_{m+n}=(a)_{m}(a+m)_{n}$.

\section{B Macaulay2 example}

In this appendix, we will give a short example using Macaulay2 [77] of the algorithm to compute fake indicial ideal for the party hat integral. The matrix $\mathrm{A}$ associated with this integral is given in eq. (3.81). Our starting point will be to compute the toric ideal $I_{\mathrm{A}}$. Using the procedure in Part II (Toric Hilbert Schemes) of ref. [57] gives

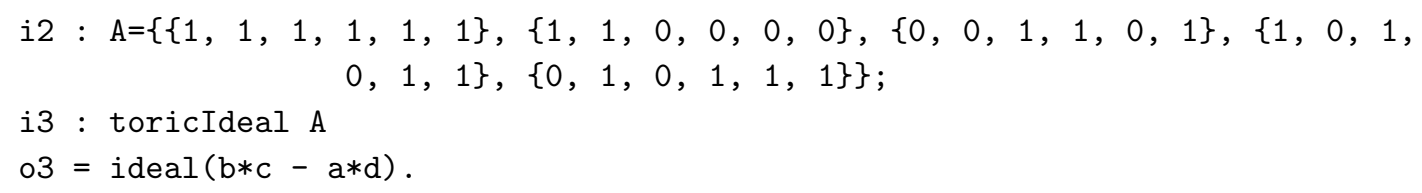


We write

$$
I_{\mathrm{A}}=\left\langle\partial_{2} \partial_{3}-\partial_{1} \partial_{4}\right\rangle \text {. }
$$

The next step it to compute the initial ideal of $I_{\mathrm{A}}$ with respect to a weight vector $w$. Using the built in Macaulay2 package $D$-modules to obtain the initial ideal with respect to $w=(0,1,1,1,1,1)$ gives

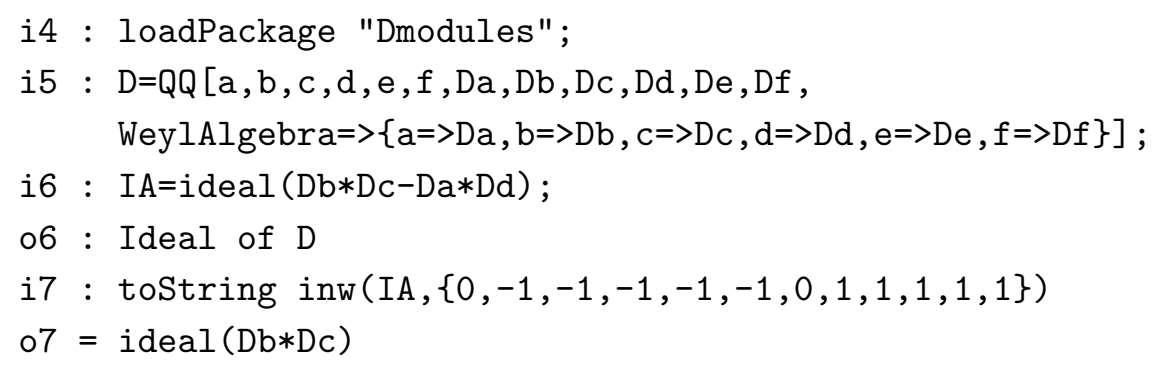

Notice that $\mathbb{K}\left[\partial_{1}, \ldots, \partial_{n}\right]$ is a commutative ring and hence $\operatorname{in}_{(-w, w)}\left(I_{A}\right)=\operatorname{in}_{w}\left(I_{A}\right)$. We have the monomial ideal

$$
\operatorname{in}_{w}\left(I_{A}\right)=\left\langle\partial_{2} \partial_{3}\right\rangle
$$

In Macaulay2 the standardPairs function computes the Standard Pairs of a monomial ideal. We define a monomial ideal in the commutative ring $\mathbb{Q}[a, \ldots, f]$ and compute its standard pairs.

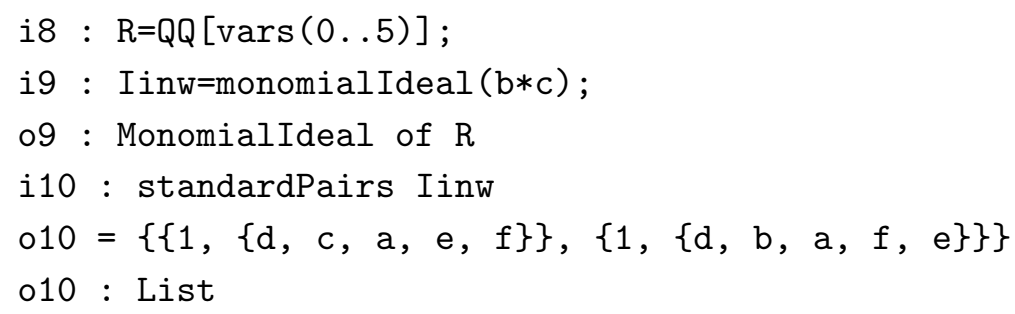

$$
\begin{aligned}
\mathcal{S}\left(\operatorname{in}_{(w)}\left(I_{A}\right)\right) & =\{\{1,\{1,3,4,5,6\}\},\{1,\{1,2,4,5,6\}\}\}, \\
\operatorname{ind}_{w}\left(I_{A}\right) & =\bigcap_{\left(\partial^{a}, K\right) \in \mathcal{S}\left(\operatorname{in}_{w}\left(I_{A}\right)\right)}\left\langle\left(\theta_{j}-a_{j}\right), j \notin K\right\rangle=\left\langle\theta_{2}\right\rangle \cap\left\langle\theta_{3}\right\rangle,
\end{aligned}
$$

therefore

$$
\operatorname{fin}_{w}\left(H_{\mathrm{A}}(\kappa)\right)=\left\langle\theta_{2} \theta_{3}\right\rangle+\left\langle A \theta-\kappa^{T}\right\rangle .
$$

Open Access. This article is distributed under the terms of the Creative Commons Attribution License (CC-BY 4.0), which permits any use, distribution and reproduction in any medium, provided the original author(s) and source are credited. 


\section{References}

[1] V. De Alfaro, B. Jaksic and T. Regge, Differential properties of Feynman amplitudes, in High-Energy Physics and Elementary Particles, Trieste Italy (1965), pg. 263.

[2] V.A. Golubeva, Some Problems in the Analytic Theory of Feynman Integrals, Russ. Math. Surv. 31 (1976) 139.

[3] A.V. Kotikov, Differential equations method: New technique for massive Feynman diagrams calculation, Phys. Lett. B 254 (1991) 158 [INSPIRE].

[4] A.V. Kotikov, Differential equation method: The Calculation of $N$ point Feynman diagrams, Phys. Lett. B 267 (1991) 123 [Erratum ibid. B 295 (1992) 409] [INSPIRE].

[5] E. Remiddi, Differential equations for Feynman graph amplitudes, Nuovo Cim. A 110 (1997) 1435 [hep-th/9711188] [INSPIRE].

[6] T. Gehrmann and E. Remiddi, Differential equations for two loop four point functions, Nucl. Phys. B 580 (2000) 485 [hep-ph/9912329] [InSPIRE].

[7] T. Gehrmann and E. Remiddi, Two loop master integrals for $\gamma * \rightarrow 3$ jets: The Planar topologies, Nucl. Phys. B 601 (2001) 248 [hep-ph/0008287] [INSPIRE].

[8] T. Gehrmann and E. Remiddi, Two loop master integrals for $\gamma * \rightarrow 3$ jets: The Nonplanar topologies, Nucl. Phys. B 601 (2001) 287 [hep-ph/0101124] [INSPIRE].

[9] J.M. Henn, Multiloop integrals in dimensional regularization made simple, Phys. Rev. Lett. 110 (2013) 251601 [arXiv:1304.1806] [INSPIRE].

[10] A. Goncharov, Geometry of configurations, polylogarithms, and motivic cohomology, Adv. Math. 114 (1995) 197.

[11] E. Remiddi and J.A.M. Vermaseren, Harmonic polylogarithms, Int. J. Mod. Phys. A 15 (2000) 725 [hep-ph/9905237] [INSPIRE].

[12] A.B. Goncharov, Multiple polylogarithms, cyclotomy and modular complexes, Math. Res. Lett. 5 (1998) 497 [arXiv:1105.2076] [INSPIRE].

[13] J. Vollinga and S. Weinzierl, Numerical evaluation of multiple polylogarithms, Comput. Phys. Commun. 167 (2005) 177 [hep-ph/0410259] [INSPIRE].

[14] V.A. Smirnov, Feynman integral calculus, Springer-Verlag, Heidelberg Germany (2006).

[15] S. Weinzierl, Introduction to Feynman Integrals, in Proceedings, Geometric and Topological Methods for Quantum Field Theory: 6th Summer School, Villa de Leyva Colombia (2009), pg. 144 [arXiv:1005.1855] [INSPIRE].

[16] J. Blumlein, S. Klein, C. Schneider and F. Stan, A Symbolic Summation Approach to Feynman Integral Calculus, arXiv:1011.2656 [INSPIRE].

[17] M. Yu. Kalmykov, Gauss hypergeometric function: Reduction, $\epsilon$-expansion for integer/half-integer parameters and Feynman diagrams, JHEP 04 (2006) 056 [hep-th/0602028] [INSPIRE].

[18] M. Yu. Kalmykov, B.F.L. Ward and S. Yost, All order $\epsilon$-expansion of Gauss hypergeometric functions with integer and half/integer values of parameters, JHEP 02 (2007) 040 [hep-th/0612240] [INSPIRE]. 
[19] M. Yu. Kalmykov, B.F.L. Ward and S.A. Yost, Multiple (inverse) binomial sums of arbitrary weight and depth and the all-order $\epsilon$-expansion of generalized hypergeometric functions with one half-integer value of parameter, JHEP 10 (2007) 048 [arXiv:0707.3654] [INSPIRE].

[20] T. Huber and D. Maître, HypExp 2, Expanding Hypergeometric Functions about Half-Integer Parameters, Comput. Phys. Commun. 178 (2008) 755 [arXiv:0708.2443] [InSPIRE].

[21] S. Moch, P. Uwer and S. Weinzierl, Nested sums, expansion of transcendental functions and multiscale multiloop integrals, J. Math. Phys. 43 (2002) 3363 [hep-ph/0110083] [INSPIRE].

[22] M. Yu. Kalmykov and B.A. Kniehl, Towards all-order Laurent expansion of generalized hypergeometric functions around rational values of parameters, Nucl. Phys. B 809 (2009) 365 [arXiv: 0807.0567] [INSPIRE].

[23] M. Yu. Kalmykov and B.A. Kniehl, Mellin-Barnes representations of Feynman diagrams, linear systems of differential equations and polynomial solutions, Phys. Lett. B 714 (2012) 103 [arXiv: 1205.1697] [INSPIRE].

[24] V.V. Bytev, M. Kalmykov, B.A. Kniehl, B.F.L. Ward and S.A. Yost, Differential Reduction Algorithms for Hypergeometric Functions Applied to Feynman Diagram Calculation, in Linear colliders. Proceedings of International Linear Collider Workshop, LCWS08 and International Linear Collider Meeting, ILC08, Chicago U.S.A. (2008) [arXiv: 0902 .1352] [INSPIRE].

[25] V.V. Bytev, M. Yu. Kalmykov and B.A. Kniehl, Differential reduction of generalized hypergeometric functions from Feynman diagrams: One-variable case, Nucl. Phys. B 836 (2010) 129 [arXiv:0904.0214] [INSPIRE].

[26] V.V. Bytev, M. Yu. Kalmykov and B.A. Kniehl, HYPERDIRE, HYPERgeometric functions DIfferential REduction: MATHEMATICA-based packages for differential reduction of generalized hypergeometric functions ${ }_{p} F_{p-1}, F_{1}, F_{2}, F_{3}, F_{4}$, Comput. Phys. Commun. 184 (2013) 2332 [arXiv: 1105.3565] [INSPIRE].

[27] M. Yu. Kalmykov and B.A. Kniehl, Counting master integrals: Integration by parts versus differential reduction, Phys. Lett. B 702 (2011) 268 [arXiv:1105.5319] [INSPIRE].

[28] S.A. Yost, V.V. Bytev, M. Yu. Kalmykov, B.A. Kniehl and B.F.L. Ward, The $\epsilon$-expansion of Feynman Diagrams via Hypergeometric Functions and Differential Reduction, arXiv: 1110.0210 [INSPIRE].

[29] V.V. Bytev and B.A. Kniehl, HYPERDIRE HYPERgeometric functions DIfferential REduction: Mathematica-based packages for the differential reduction of generalized hypergeometric functions: Horn-type hypergeometric functions of two variables, Comput. Phys. Commun. 189 (2015) 128 [arXiv: 1309.2806] [INSPIRE].

[30] V.V. Bytev and B.A. Kniehl, HYPERDIRE - HYPERgeometric functions DIfferential REduction: Mathematica-based packages for the differential reduction of generalized hypergeometric functions: Lauricella function Fc of three variables, Comput. Phys. Commun. 206 (2016) 78 [arXiv:1602.00917] [INSPIRE].

[31] N. Takayama, Gröbner basis and the problem of contiguous relation, Japan J. Appl. Math. 6 (1989) 147.

[32] N. Takayama, Algorithm finding recurrence relations of binomial sums and its complexity, J. Symb. Comput. 20 (1995) 637. 
[33] M. Yu. Kalmykov, V.V. Bytev, B.A. Kniehl, B.F.L. Ward and S.A. Yost, Feynman Diagrams, Differential Reduction and Hypergeometric Functions, PoS (ACAT08) 125 (2008) [arXiv:0901.4716] [INSPIRE].

[34] M. Yu. Kalmykov and B.A. Kniehl, Counting the number of master integrals for sunrise diagrams via the Mellin-Barnes representation, JHEP 07 (2017) 031 [arXiv:1612.06637] [INSPIRE].

[35] I. Gelfand, M. Kapranov and A. Zelevinsky, Generalized Euler integrals and A-hypergeometric functions, Adv. Math. 84 (1990) 255.

[36] I.M. Gelfand, M.M. Kapranov and A. Zelevinsky, Discriminants, resultants and multidimensional determinants, Birkhäuser, Boston U.S.A. (1994).

[37] L. Nilsson and M. Passare, Mellin transforms of multivariate rational functions, J. Geom. Anal. 23 (2010) 24 [arXiv:1010.5060].

[38] C. Berkesch, J. Forsgård and M. Passare, Euler-Mellin integrals and A-hypergeometric functions, Michigan Math. J. 1 (2014) 101 [arXiv:1103.6273].

[39] J. Forsgård, L.F. Matusevich and A. Sobieska, On transformations of A-hypergeometric functions, arXiv:1703.03036.

[40] M. Saito, B. Sturmfels, and N. Takayama, Groebner Deformations of Hypergeometric Differential Equations, Graduate texts in mathematics, Springer-Verlag, Heidelberg Germany (1999).

[41] E. Nasrollahpoursamami, Periods of Feynman Diagrams and GKZ D-Modules, arXiv: 1605.04970.

[42] K. Schultka, Toric geometry and regularization of Feynman integrals, arXiv:1806.01086 [INSPIRE].

[43] P. Vanhove, Feynman integrals, toric geometry and mirror symmetry, in Proceedings, KMPB Conference: Elliptic Integrals, Elliptic Functions and Modular Forms in Quantum Field Theory, Zeuthen Germany (2017), pg. 415 [arXiv:1807.11466] [INSPIRE].

[44] R.N. Lee and A.A. Pomeransky, Critical points and number of master integrals, JHEP 11 (2013) 165 [arXiv: 1308.6676] [inSPIRE].

[45] E. Cattani, Three Lectures on Hypergeometric Functions, Lecture notes (2006).

[46] J. Forsgård, On hypersurface coamoebas and integral representations of A-hypergeometric functions, Licentiate Thesis, Stockholm University, Stockholm Sweden (2012).

[47] J. Stienstra, GKZ hypergeometric structures, in Instanbul 2005: CIMPA Summer School on Arithmetic and Geometry Around Hypergeometric Functions, Istanbul Turkey (2005) [math/0511351].

[48] B. Sturmfels, Groebner Bases and Convex polytopes, University Lecture Series, AMS Press, New York U.S.A. (1996).

[49] G. Ziegler, Graduate Texts in Mathematics. Vol. 152: Lectures on Polytopes, Springer-Verlag, New York U.S.A. (1994).

[50] B. Feng, Y.-H. He, K.D. Kennaway and C. Vafa, Dimer models from mirror symmetry and quivering amoebae, Adv. Theor. Math. Phys. 12 (2008) 489 [hep-th/0511287] [INSPIRE].

[51] J. Forsgård, On dimer models and coamoebas, Ann. Henri Poincaré D 6 (2019) 199 [arXiv: 1602.01826]. 
[52] P. Johansson, Coamoebas, Licentiate Thesis, Stockholm University, Stockholm Sweden (2010).

[53] T. Theobald and T. de Wolff, Approximating amoebas and coamoebas by sums of squares, Math. Comp. 84 (2015) 455 [arXiv:1101.4114].

[54] J. Forsgård and P. Johansson, On the order map for hypersurface coamoebas, Ark. Mat. 53 (2015) 79 [arXiv:1205.2014].

[55] J. Forsgård, L.F. Matusevich, N. Mehlhop and T. de Wolff, Lopsided Approximation of Amoebas, Math. Comp. 88 (2019) 485 [arXiv:1608.08663].

[56] J. Forsgård, Tropical aspects of real polynomials and hypergeometric functions, Ph.D. Thesis, Stockholm University, Stockholm Sweden (2014).

[57] D. Eisenbud, D.R. Grayson, M. Stillman and B. Sturmfels, Computations in algebraic geometry with Macaulay 2, Algorithms and computations in mathematics, Springer-Verlag, Heidelberg Germany (2001).

[58] C. Bogner and S. Weinzierl, Resolution of singularities for multi-loop integrals, Comput. Phys. Commun. 178 (2008) 596 [arXiv:0709.4092] [INSPIRE].

[59] C. Bogner and S. Weinzierl, Feynman graph polynomials, Int. J. Mod. Phys. A 25 (2010) 2585 [arXiv: 1002.3458] [INSPIRE].

[60] S. Weinzierl, Feynman Graphs, in Computer Algebra in Quantum Field Theory. Integration, Summation and Special Functions. Proceedings of LHCPhenoNet School, Linz Austria (2012), pg. 381 [arXiv: 1301.6918] [INSPIRE].

[61] C. Berkesch Zamaere, J. Forsgård and L.F. Matusevich, On the parametric behavior of A-hypergeometric series, Trans. Am. Math. Soc. 370 (2018) 4089 [arXiv:1603.08954].

[62] C. Anastasiou, E.W.N. Glover and C. Oleari, Scalar one loop integrals using the negative dimension approach, Nucl. Phys. B 572 (2000) 307 [hep-ph/9907494] [INSPIRE].

[63] O.V. Tarasov, Massless on-shell box integral with arbitrary powers of propagators, J. Phys. A 51 (2018) 275401 [arXiv: 1709.07526] [INSPIRE].

[64] O.V. Tarasov, A New approach to the momentum expansion of multiloop Feynman diagrams, Nucl. Phys. B 480 (1996) 397 [hep-ph/9606238] [INSPIRE].

[65] O.V. Tarasov, Reduction of Feynman graph amplitudes to a minimal set of basic integrals, Acta Phys. Polon. B 29 (1998) 2655 [hep-ph/9812250] [INSPIRE].

[66] C. Anastasiou, E.W.N. Glover and C. Oleari, Application of the negative dimension approach to massless scalar box integrals, Nucl. Phys. B 565 (2000) 445 [hep-ph/9907523] [INSPIRE].

[67] E.E. Boos and A.I. Davydychev, A Method of the Evaluation of the Vertex Type Feynman Integrals, Moscow Univ. Phys. Bull. 42N3 (1987) 6 [Vestn. Mosk. Univ. Fiz. Astron. 28N3 (1987) 8].

[68] E.E. Boos and A.I. Davydychev, A Method of evaluating massive Feynman integrals, Theor. Math. Phys. 89 (1991) 1052 [INSPIRE].

[69] T. Bitoun, C. Bogner, R.P. Klausen and E. Panzer, Feynman integral relations from parametric annihilators, Lett. Math. Phys. 109 (2019) 497 [arXiv:1712.09215] [INSPIRE].

[70] P.A. Baikov, Explicit solutions of the multiloop integral recurrence relations and its application, Nucl. Instrum. Meth. A 389 (1997) 347 [hep-ph/9611449] [INSPIRE]. 
[71] M. Harley, F. Moriello and R.M. Schabinger, Baikov-Lee Representations Of Cut Feynman Integrals, JHEP 06 (2017) 049 [arXiv: 1705.03478] [INSPIRE].

[72] H. Frellesvig and C.G. Papadopoulos, Cuts of Feynman Integrals in Baikov representation, JHEP 04 (2017) 083 [arXiv:1701.07356] [InSPIRE].

[73] H. Frellesvig et al., Decomposition of Feynman Integrals on the Maximal Cut by Intersection Numbers, JHEP 05 (2019) 153 [arXiv:1901.11510] [INSPIRE].

[74] J. Bosma, M. Sogaard and Y. Zhang, Maximal Cuts in Arbitrary Dimension, JHEP 08 (2017) 051 [arXiv: 1704. 04255] [INSPIRE].

[75] P. Mastrolia and S. Mizera, Feynman Integrals and Intersection Theory, JHEP 02 (2019) 139 [arXiv: 1810.03818] [INSPIRE].

[76] T. Hibi, K. Nishiyama and N. Takayama, Pfaffian systems of A-hypergeometric equations I: Bases of twisted cohomology groups, Adv. Math. 306 (2017) 303 [arXiv:1212.6103].

[77] D.R. Grayson and M.E. Stillman, Macaulay2, a software system for research in algebraic geometry, available at http://www.math.uiuc.edu/Macaulay2/. 